\title{
JORDAN HOMOMORPHISMS OF RINGS
}

\author{
BY \\ N. JACOBSON AND C. E. RICKART
}

The primary aim of this paper is to study mappings $J$ of rings that are additive and that satisfy the conditions

$$
\left(a^{2}\right)^{J}=\left(a^{J}\right)^{2}, \quad(a b a)^{J}=a^{J} b^{J} a^{J} .
$$

Such mappings will be called Jordan homomorphisms. If the additive groups admit the operator $1 / 2$ in the sense that $2 x=a$ has a unique solution (1/2) $a$ for every $a$, then conditions (1) are equivalent to the simpler condition

$$
(a b)^{J}+(b a)^{J}=a^{J} b^{J}+b^{J} a^{J} .
$$

Mappings satisfying (2) were first considered by Ancochea [1], [2](1). The modification to (1) is essentially due to Kaplansky [13]. Its purpose is to obviate the necessity of imposing any restriction on the additive groups of the rings under consideration.

If $\mathfrak{A}$ is a ring, it is customary to define the Jordan ring $\mathfrak{A}_{j}$ to be the system obtained from $\mathfrak{A}$ by replacing ordinary multiplication by Jordan multiplication $\{a b\}=a b+b a$. Then it is clear that $J$ is an additive mapping of the ring $\mathfrak{A}$ into a ring $\mathfrak{B}$ satisfying (2) if and only if $J$ is a homomorphism of the Jordan ring $\mathfrak{A}_{j}$ into the Jordan ring $\mathfrak{B}_{j}$. The replacement of (2) by (1) now suggests the following modification in the definition of $\mathfrak{A}_{j}: \mathfrak{A}_{j}$ is the system consisting of the set $\mathfrak{A}$, the addition + , the unary composition $a \rightarrow a^{2}$, and the binary composition $a \rightarrow a b a$. More generally we are led to define a special Jordan ring to be a subset of a ring that is a subgroup under + and that is closed under the compositions $a \rightarrow a^{2}, a \rightarrow a b a$. In these terms the problem that we are considering is that of determining the homomorphisms of special Jordan rings $\mathfrak{A}_{j}$ into special Jordan rings $\mathfrak{B}_{j}$. In a subsequent paper we hope to consider the homomorphisms of the special Jordan rings of symmetric elements relative to involutions in rings.

It is immediate that any (associative) homomorphism or anti-homomorphism of a ring $\mathfrak{A}$ is a Jordan homomorphism. Also if $J_{i}, i=1,2$, is a Jordan homomorphism of $\mathfrak{A}$ into $\mathfrak{B}_{i}$, then the mapping $a \rightarrow a^{J_{1}}+a^{J_{2}}$ of $\mathfrak{A}$ into $\mathfrak{B}_{1} \oplus \mathfrak{B}_{2}$ is a Jordan homomorphism. We call this mapping the sum $J_{1}+J_{2}$ of $J_{1}$ and $J_{2}$. Our main results give sufficient conditions that Jordan homomorphisms be either homomorphisms or anti-homomorphisms or sums of homomorphisms and anti-homomorphisms. Thus we prove the following

Presented to the society, September 2, 1949; received by the editors January 3, 1950 and, in revised form, May 8, 1950.

(1) Numbers in brackets refer to the bibliography at the end of the paper. 
extension of a recent result of Hua [5]: Any Jordan homomorphism of an arbitrary ring into an integral domain is either a homomorphism or an antihomomorphism.

One of the main results which we obtain is that if $\mathfrak{A}$ is a matrix ring $\mathfrak{D}_{n}$, $n \geqq 2, \mathfrak{D}$ an arbitrary ring with an identity, then any Jordan homomorphism of $\mathfrak{A}$ is the sum of a homomorphism and an anti-homomorphism. We also extend this result to an extensive class of rings which are locally matrix in the sense that every finite subset can be embedded in a matrix subring $\mathfrak{D}_{n}$, $n \geqq 2$. By a result of Litoff any simple ring with minimal one-sided ideals is either a division ring or a locally matrix ring. It follows that our theorem applies to these rings. The present method also yields a determination of the Jordan automorphisms of primitive rings with minimal ideals. In this connection we have had to obtain information on the ideal structure of the Jordan rings obtained from primitive rings with minimal ideals.

The second main method which we have developed for studying Jordan homomorphisms is a Lie ring method. The starting point here is the observation that any Jordan homomorphism $J$ satisfies the condition

$$
[[a b] c]^{J}=\left[\left[a^{J} b^{J}\right] c^{J}\right],
$$

where as usual $[x y]$ denotes $x y-y x$. An additive mapping satisfying this condition is called a Lie triple system homomorphism. We have been led to determine conditions that such mappings be Lie ring homomorphisms or antihomomorphisms. For these conditions together with the Jordan homomorphism condition give conditions that a Jordan homomorphism be an associative homomorphism or anti-homomorphism. The conditions which we obtain deal mainly with the ideal structure of the Lie rings determined by the given rings. In order to apply our results to primitive rings, we have had to investigate the Lie ring structure of rings of this type.

We note finally that our results are also applicable to the theory of derivations. Thus, they give conditions that a Jordan derivation of a ring, that is, an additive mapping $D$ of a ring into itself such that

$$
\left(a^{2}\right)^{D}=a a^{D}+a^{D} a, \quad(a b a)^{D}=a^{D} b a+a b^{D} a+a b a^{D},
$$

be an ordinary derivation.

1. Definitions and elementary properties. A special Jordan ring $J$ is a subset of an associative ring that is a subgroup under + and that contains $a^{2}$ and $a b a$ for every $a, b$ in $\Im$. A special Lie ring $\mathfrak{R}$ is a subset of an associative ring that is a subgroup under + and that contains $[a b]=a b-b a$ for every $a, b$ in $\mathfrak{R}$. A Lie triple system $\mathfrak{U}$ is a subset of an associative ring that is a subgroup under + and that contains $[[a b] c]$ for every $a, b, c$ in $\mathfrak{U}$.

We are primarily interested in this paper in special Jordan rings and their homomorphisms. A mapping $J$ of a special Jordan ring $\Im$ into a special Jordan ring $\mathfrak{B}$ is a homomorphism if 


$$
\begin{gathered}
(a+b)^{J}=a^{J}+b^{J}, \\
\left(a^{2}\right)^{J}=\left(a^{J}\right)^{2}, \quad(a b a)^{J}=a^{J} b^{J} a^{J} .
\end{gathered}
$$

If $\Im=\mathscr{A}$ is a ring, then we shall also say that $J$ is a Jordan homomorphism of the associative ring $\mathfrak{A}$.

If $\Im$ is a subgroup of the additive group of a ring that admits the operator $1 / 2$, then $\Im$ is a special Jordan ring if and only if $\Im$ is closed under the composition $\{a b\}=a b+b a$. For if $\Im$ is a special Jordan ring, then certainly $\{a b\}=(a+b)^{2}-a^{2}-b^{2}$ is in $\Im$. On the other hand, if $\Im$ is closed under $\{a b\}$ then $\Im$ contains $a^{2}=(1 / 2)\{a a\}$ and $a^{3}=(1 / 2)\left\{a^{2} a\right\}$. Hence $\Im$ contains

$$
a b a+b a b=(a+b)^{3}-a^{3}-b^{3}-a^{2} b-b a^{2}-b^{2} a-a b^{2} .
$$

If we replace $b$ by $-b$, we see that $I$ contains $-a b a+b a b$. It follows that $I$ contains $b a b$. In a similar fashion we can see that an additive mapping of a special Jordan ring $\Im$ into a special Jordan ring $\Im^{J}$ that has no elements of additive order 2 is a Jordan homomorphism if and only if $(a b)^{J}+(b a)^{J}$ $=a^{J} b^{J}+b^{J} a^{J}$.

An element $u$ of a ring that can be obtained from a set of elements $a_{1}, a_{2}, \cdots, a_{n}$ by performing the operations of addition, subtraction, $a \rightarrow a^{2}$, $a \rightarrow a b a$ will be called a Jordan polynomial in the $a_{i}$. If the $a_{i}$ belong to the special Jordan ring $\Im$, then so does $u$, and if $J$ is a homomorphism of $\Im$, then $J$ maps $u$ into the element obtained from the $a_{i}^{J}$ in the same manner as $u$ is obtained from the $a_{i}$. Important examples of Jordan polynomials are

$$
\begin{aligned}
a b+b a & =(a+b)^{2}-a^{2}-b^{2}, \\
a b c+c b a & =(a+c) b(a+c)-a b a-c b c, \\
{[[a b] c] } & =a b c+c b a-(b a c+c a b), \\
{[a b]^{2} } & =a(b a b)+(b a b) a-a b^{2} a-b a^{2} b .
\end{aligned}
$$

Also the powers of $a$ are Jordan polynomials. For by definition $a^{2}$ and $a^{3}=a a a$ are Jordan polynomials and if $a^{r-1}, r \geqq 3$, is a Jordan polynomial, then so is $a^{r+1}=a a^{r-1} a$. Since $[[a b] c]$ is a Jordan polynomial, we see that any special Jordan ring is a Lie triple system. Also, it is now clear that if $J$ is a Jordan homomorphism, then $\left(a^{n}\right)^{J}=\left(a^{J}\right)^{n}, \quad[[a b] c]^{J}=\left[\left[a^{J} b^{J}\right] c^{J}\right]$, and $\left([a b]^{2}\right)^{J}$ $=\left[a^{J} b^{J}\right]^{2}$, that is, we have the following result.

Theorem 1. Any Jordan homomorphism preserves arbitrary powers, Lie triple products, and squares of commutators.

COROLLARY 1. If $[a b]=0$ and the enveloping associative ring $\left({ }^{2}\right)$ of $\mathfrak{A}^{J}$ has no nonzero nilpotent elements in its center, then $\left[a^{J} b^{J}\right]=0$.

Proof. $[a b]=0$ implies $[[a b] x]=0$ for all $x$ in $\mathfrak{A}$. Hence $\left[\left[a^{J} b^{J}\right] x^{J}\right]=0$ so

(2) That is, the associative ring generated by the set $\mathfrak{Q}^{J}$. 
that $\left[a^{J} b^{J}\right]$ is in the center of the enveloping ring of $\mathfrak{P}^{J}$. Moreover, $[a b]^{2}=0$; hence $\left[a^{J} b^{J}\right]^{2}=0$. Hence, $\left[a^{J} b^{J}\right]=0$.

CoRollary 2. If $e$ is an idempotent and $a$ is an element such that $[e a]=0$, then $(e a)^{J}=e^{J} a^{J}=a^{J} e^{J}$. If $e a=a e=a$, then $e^{J} a^{J}=a^{J} e^{J}=a^{J}$ and if $e$ and $a$ are orthogonal (ea=ae=0), then so are $e^{J}$ and $a^{J}$.

Proof. By Theorem 1, $\left[e^{J} a^{J}-a^{J} e^{J}, e^{J}\right]=0$. Hence $e^{J} a^{J}-e^{J} a^{J} e^{J}=e^{J} a^{J} e^{J}$ $-a^{J} e^{J}$. Left multiplication by $e^{J}$ gives $e^{J} a^{J}=e^{J} a^{J} e^{J}$; right multiplication by $e^{J}$ gives $a^{J} e^{J}=e^{J} a^{J} e^{J}$. Also $e^{J} a^{J} e^{J}=(e a e)^{J}=(e a)^{J}=(a e)^{J}$. Hence $(e a)^{J}=e^{J} a^{J}=a^{J} e^{J}$. The remaining statements are obvious consequences.

CoROLlaRy 3. If $\mathfrak{A}$ has an identiy 1 , then $1^{J}$ is an identity for the enveloping ring of $\mathfrak{A}^{J}$. If a is a unit, so is $a^{J}$.

Proof. The first statement is contained in Corollary 2. Now suppose that $a b=1=b a$. Then $a b^{2} a=1$ and $a^{J}\left(b^{J}\right)^{2} a^{J}=1^{J}$. Hence $a^{J}$ is a unit.

We note next a fundamental identity for Jordan homomorphisms of rings. This is the relation

$$
\left[(a b)^{J}-a^{J} b^{J}\right]\left[(a b)^{J}-b^{J} a^{J}\right]=0
$$

which holds since

$$
\begin{aligned}
{\left[(a b)^{J}-a^{J} b^{J}\right]\left[(a b)^{J}-b^{J} a^{J}\right] } & =(a b)^{J}(a b)^{J}-(a b)^{J} b^{J} a^{J}-a^{J} b^{J}(a b)^{J}+a^{J}\left(b^{J}\right)^{2} a^{J} \\
& =(a b a b)^{J}-((a b) b a+a b(a b))^{J}+a^{J}\left(b^{J}\right)^{2} a^{J} \\
& =\left(a b a b-a b^{2} a-a b a b+a b^{2} a\right)^{J}=0 .
\end{aligned}
$$

Similarly we can verify that

$$
\begin{aligned}
{\left[(a b)^{J}-b^{J} a^{J}\right]\left[(a b)^{J}-a^{J} b^{J}\right] } & =0, \\
{\left[(a b c)^{J}-a^{J} b^{J} c^{J}\right] b^{J}\left[(a b c)^{J}-c^{J} b^{J} a^{J}\right] } & =0, \\
{\left[(a b c)^{J}-c^{J} b^{J} a^{J}\right] b^{J}\left[(a b c)^{J}-a^{J} b^{J} c^{J}\right] } & =0 .
\end{aligned}
$$

2. Jordan homomorphisms into integral domains. In this section we prove a generalization of Hua's theorem [5] that a Jordan automorphism of a division ring is either an automorphism or an anti-automorphism.

TheOREM 2. If $J$ is a Jordan homomorphism of a ring $\mathfrak{A}$ into an integral domain $\mathfrak{B}$, then $J$ is either a homomorphism or an anti-homomorphism.

Proof. By the identity (7), for any $a, b$ in $\mathfrak{A}$ we have either $(a b)^{J}=a^{J} b^{J}$ or $(a b)^{J}=b^{J} a^{J}$. Our result is therefore an immediate consequence of the following lemma which is due to Hua:

LEMMA 1. Let $\mathfrak{A}$ be an arbitrary distributive system (nonassociative ring) and let $J$ be an additive mapping of $\mathfrak{A}$ into a second distributive system $\mathfrak{B}$ such that for every pair $a, b$ in $\mathfrak{A}$ either $(a b)^{J}=a^{J} b^{J}$ or $(a b)^{J}=b^{J} a^{J}$. Then $J$ is either $a$ 
homomorphism or an anti-homomorphism.

Proof. If both $(a b)^{J}=a^{J} b^{J}$ and $(a b)^{J}=b^{J} a^{J}$ hold for all $a$ and $b$, then $J$ is both a homomorphism and an anti-homomorphism. Now suppose that $a, b$ is a pair for which $(a b)^{J}=a^{J} b^{J} \neq b^{J} a^{J}$. If $d$ is any other element, then $(a d)^{J}$ $=a^{J} d^{J}$. For otherwise $(a d)^{J}=d^{J} a^{J} \neq a^{J} d^{J}$ and these relations are incompatible with

$$
(a(b+d))^{J}=\left\{\begin{array}{l}
a^{J} b^{J}+a^{J} d^{J}, \\
b^{J} a^{J}+d^{J} a^{J} .
\end{array}\right.
$$

Similarly $(c b)^{J}=c^{J} b^{J}$ for any $c$. Next let $c$ and $d$ be elements such that $(c d)^{J}$ $=d^{J} c^{J} \neq c^{J} d^{J}$. Then a similar argument shows that $(a d)^{J}=d^{J} a^{J}$ and $(c b)^{J}$ $=b^{J} c^{J}$ hold. Now, if

$$
\begin{aligned}
((a+c)(b+d))^{J} & =\left(a^{J}+c^{J}\right)\left(b^{J}+d^{J}\right) \\
& =a^{J} b^{r}+a^{J} d^{J}+c^{J} b^{J}+c^{J} d^{J},
\end{aligned}
$$

then $(c d)^{J}=c^{J} d^{J}$ contrary to assumption. Similarly

$$
((a+c)(b+d))^{J}=\left(b^{J}+d^{J}\right)\left(a^{J}+c^{J}\right)
$$

leads to the contradiction $(a b)^{J}=b^{J} a^{J}$. Hence the condition $(a b)^{J} \neq b^{J} a^{J}$ and $(c d)^{J} \neq c^{J} d^{J}$ are incompatible.

The following examples show that we cannot assert that Jordan homomorphisms of integral domains or of fields are always homcmorphisms or anti-homomorphisms.

EXAMPLE 1. Let $\mathfrak{A}=\Phi[x, y]$ be the polynomial ring in two independent indeterminates over a field $\Phi$ of characteristic $\neq 2$. The elements $x^{k} y^{l}, k, l$ $=0,1,2, \cdots$, form a basis and we have the Jordan multiplication table

$$
\left\{x^{k} y^{l}, x^{m} y^{n}\right\}=2 x^{k+m} y^{l+n} \text {. }
$$

Let $\mathfrak{B}=\Phi[X, Y, Z]$ be the polynomial ring in the three elements $X, Y, Z$ that satisfy the relations

$$
Y X=X Y+Z, \quad[X Z]=[Y Z]=Z^{2}=0 .
$$

Then

$$
Y^{l} X^{k}=X^{k} Y^{l}+k l X^{k-1} Y^{l-1} Z,
$$

and $(1 / 2)\left\{X^{k} Y^{l}\right\}=X^{k} Y^{l}+(k l / 2) X^{k-1} Y^{l-1} Z$. By direct calculation one verifies that

$$
\left\{(1 / 2)\left\{X^{k} Y^{l}\right\},(1 / 2)\left\{X^{m} Y^{n}\right\}\right\}=\left\{X^{k+m} \dot{Y}^{l+n}\right\} .
$$

Hence the linear mapping that sends $x^{k} y^{l}$ into $(1 / 2)\left\{X^{k} Y^{l}\right\}$ is a Jordan homomorphism. Since $\left((1 / 2)\left\{X^{k} Y^{l}\right\}\right)\left((1 / 2)\left\{X^{m} Y^{n}\right\}\right) \neq(1 / 2)\left\{X^{k+m} Y^{l+n}\right\}$, the mapping is neither a homomorphism nor an anti-homomorphism. 
ExAMPLE 2. Let $\mathfrak{A}$ be a purely inseparable field of the form $\Phi(x, y)$ where $x^{p}=\xi, y^{p}=\eta$, and the elements $x^{i} y^{j}, i, j=0,1, \cdots, p-1$, form a basis. Let $\mathfrak{B}$ be the algebra over $\Phi$ generated by $X, Y, Z$ such that

$$
\begin{gathered}
Y X=X Y+Z, \quad[X Z]=[Y Z]=Z^{2}=0, \\
X^{p}=\xi, \quad Y^{p}=\eta .
\end{gathered}
$$

If $p \neq 2$ we can verify as in example 1 that the linear mapping which sends $x^{k} y^{l}$ into $(1 / 2)\left\{X^{k} Y^{l}\right\}$ is a Jordan homomorphism that is not a homomorphism.

3. Sums of Jordan homomorphisms. In this section we shall obtain some general criteria that a Jordan homomorphism of a ring be a sum of a homomorphism and an anti-homomorphism. If $J_{i}$ is a Jordan homomorphism of $\mathfrak{A}$ into $\mathfrak{B}_{i}$, we have defined the sum $J=J_{1}+J_{2}$ to be the mapping $a \rightarrow a^{J_{1}}+a^{J_{2}}$ into $\mathfrak{B}_{1} \oplus \mathfrak{B}_{2}$. If $\mathfrak{A}^{\prime}$ is anti-isomorphic to $\mathfrak{A}$ under the correspondence $a \rightarrow a^{\prime}$, then the sum mapping $a \rightarrow \bar{a} \equiv a+a^{\prime}$ of $\mathfrak{A}$ into $\mathfrak{A} \oplus \mathfrak{A}^{\prime}$ will be called a symmetrized direct sum mapping of $\mathfrak{R}$.

Now let $J$ and $K$ be any two Jordan homomorphisms. Then we shall say that $J$ is a cover of $K$ (or $J$ covers $K$ ) if the correspondence $a^{J} \rightarrow a^{K}$ can be extended to a homomorphism of the enveloping ring $\mathbb{F}_{J}$ of the elements $a^{J}$ onto the enveloping ring $\mathbb{E}_{K}$ of the $a^{K}$. Evidently if such an extension exists, it is unique. The elements of $\mathcal{F}_{J}$ are polynomials with integral coefficients in the elements $a^{J}$. It follows easily from this remark that $J$ is a cover of $K$ if and only if any polynomial relation $P\left(a_{1}^{J}, a_{2}^{J}, \cdots, a_{r}^{J}\right)=0$ implies the corresponding relation $P\left(a_{1}^{K}, a_{2}^{K}, \cdots, a_{r}^{K}\right)=0$. Since $P\left(\bar{a}_{1}, \bar{a}_{2}, \cdots, \bar{a}_{r}\right)$ $=P\left(\bar{a}_{1}, \bar{a}_{2}, \cdots, \bar{a}_{r}\right)+P\left(a_{1}^{\prime}, a_{2}^{\prime}, \cdots, a_{r}^{\prime}\right)$ for $\bar{a}_{i}=a_{i}+a_{i}^{\prime}$, as above, $P\left(a_{1}, a_{2}, \cdots, a_{r}\right)=0$ is equivalent to the two conditions

$$
P\left(a_{1}, a_{2}, \cdots, a_{r}\right)=0, \quad P\left(a_{1}^{\prime}, a_{2}^{\prime}, \cdots, a_{r}^{\prime}\right)=0 .
$$

If $P_{1}\left(a_{1}, a_{2}, \cdots, a_{r}\right)$ denotes the polynomial which is obtained from $P$ by reversing the order of the terms in each monomial, then the condition $P\left(a_{1}^{\prime}, a_{2}^{\prime}, \cdots, a_{r}^{\prime}\right)=0$ is equivalent to $P_{1}\left(a_{1}, a_{2}, \cdots, a_{r}\right)=0$. Hence a necessary and sufficient condition that $K$ be covered by the symmetrized direct sum mapping is that for any pair of relations

$$
P\left(a_{1}, a_{2}, \cdots, a_{r}\right)=0, \quad P_{1}\left(a_{1}, a_{2}, \cdots, a_{r}\right)=0
$$

in $\mathfrak{A}$ we have the corresponding relation $P\left(a_{1}^{K}, a_{2}^{K}, \cdots, a_{r}^{K}\right)=0$ in the image ring.

Since the conditions that $J$ cover $K$ are of "finite character," we evidently have the following result.

Theorem 3. Let $\mathfrak{A}$ be a ring, $J$ and $K$ Jordan homomorphisms. Assume that every finite subset $F$ of $\mathfrak{A}$ is contained in a subring $\mathfrak{A}_{F}$ such that the induced mapping $J$ of $\mathfrak{A}_{F}$ is a cover of the induced mapping $K$; then $J$ is a cover of $K$. 
It is clear that $J=J_{1}+J_{2}$ covers $J_{1}$ and $J_{2}$ and that if $K$ is a cover of $J_{1}$ and $J_{2}$, then $K$ is a cover of $J_{1}+J_{2}$. Also if $J_{i}$ covers $K_{i}$ for $i=1,2$, then $J_{1}+J_{2}$ covers $K_{1}+K_{2}$. In particular, it is clear that the symmetrized direct sum mapping covers any $J_{1}+J_{2}$ where $J_{1}$ is a homomorphism and $J_{2}$ is an anti-homomorphism. A partial converse of this result is given in the following theorem.

THEOREM 4. Let $\mathfrak{A}$ be a ring with the following properties: (1) The enveloping ring of the elements $\bar{a}=a+a^{\prime}$ in $\mathfrak{S}=\mathfrak{A} \oplus \mathfrak{A}^{\prime}$ is $\mathfrak{S}$, (2) Every ideal in $\mathfrak{S}$ has the form $\Omega_{1} \oplus \Omega_{2}$ where $\Omega_{1}$ is an ideal in $\mathfrak{A}$ and $\Omega_{2}$ is an ideal in $\mathfrak{H}^{\prime}$. Then any Jordan homomorphism $J$ that is covered by the symmetrized direct sum mapping is a sum of a homomorphism and an anti-homomorphism.

Proof. Let $U$ denote the homomorphism that maps $\bar{a}$ into $a^{J}$. Since the enveloping ring of the $\bar{a}$ is $\subseteq, U$ is a homomorphism of $\subseteq$ onto the enveloping ring $\mathfrak{E}$ of $\mathfrak{A}^{J}$. Evidently, $\mathfrak{E}_{1}=\mathfrak{U}^{U}$ and $\mathfrak{F}_{2}=\mathfrak{Y}^{\prime} U$ are ideals in $\mathbb{E}$. If $\Re$ is the kernel of $U$, then the inverse image of $\mathfrak{E}_{1}$ is $\mathfrak{U}+\Re$ while that of $\mathfrak{F}_{2}$ is $\mathfrak{U}^{\prime}+\Re$. Since $\mathfrak{R}=\Omega_{1} \oplus \Omega_{2}$ where $\Omega_{1} \subseteq \mathfrak{A}$ and $\Omega_{2} \subseteq \mathfrak{A}^{\prime}, \mathfrak{A}+\mathfrak{\Omega}=\mathfrak{A}+\Omega_{2}$, and $\mathfrak{A}^{\prime}+\mathfrak{R}=\mathfrak{A}^{\prime}+\Omega_{1}$. Hence the inverse image of $\mathfrak{F}_{1} \cap \mathfrak{F}_{2}$ is

$$
(\mathfrak{A}+\Re) \cap\left(\mathfrak{U}^{\prime}+\Re\right)=\left(\mathfrak{U}+\Re_{2}\right) \cap\left(\mathfrak{U}^{\prime}+\Re_{1}\right)=\Re .
$$

Hence $\mathfrak{F}_{1} \cap \mathfrak{F}_{2}=0$ and $\mathfrak{F}_{=}=\mathfrak{F}_{1} \oplus \mathfrak{F}_{2}$. Evidently the mapping $a \rightarrow a^{U}$ is a homomorphism $J_{1}$ of $\mathfrak{A} \subseteq \subseteq$ and $a \rightarrow a^{\prime} \equiv a^{J_{2}}$ is an anti-homomorphism. Since $a^{J}=\bar{a}^{U}=a^{U}+a^{U}=a^{J_{1}}+a^{J_{2}}$ and $a^{J_{i}} \in \xi_{i}, J=J_{1}+J_{2}$.

The following two theorems give simple sufficient conditions that the hypotheses of Theorem 4 hold.

THEOREM 5. If $\mathfrak{A}$ is a ring such that the smallest ideal containing all the elements $[a b]$ is $\mathfrak{A}$, then the enveloping ring of the elements $\bar{a}=a+a^{\prime}$ in $\mathfrak{S}=\mathfrak{A} \oplus \mathfrak{A}^{\prime}$ is $\mathfrak{s}$.

Proof. Let $\overline{\mathfrak{S}}$ be the enveloping ring of the $\bar{a}$. Then $\overline{\mathfrak{S}}$ contains $\bar{a} \bar{b}-\overline{b a}$ $=[a b]$. Also $\widetilde{\mathfrak{S}}$ contains $[a b] \bar{c}=[a b] c$ and $\bar{c}[a b]=c[a b]$ for any $c$ in $\mathfrak{A}$. In this way we can see that $\overline{\mathfrak{S}}$ contains the ideal generated by the elements $[a b]$. Hence $\overline{\mathfrak{S}} \supseteq \mathfrak{A}$. It follows also that $\overline{\mathfrak{S}} \supseteq \mathfrak{H}^{\prime}$. Hence $\overline{\mathfrak{S}}=\mathfrak{\Im}$.

ThEOREM 6. A sufficient condition that every ideal $\mathfrak{\Omega}$ in $\mathfrak{S}=\mathfrak{A} \oplus \mathfrak{U}^{\prime}$ have the form $\Omega_{1} \oplus \Omega_{2}$ where $\Re_{1}=\mathfrak{A} \cap \Re$ and $\mathfrak{\Omega}_{2}=\mathfrak{X} \cap \cap \Omega_{\text {is }}$ that for every $u \in \mathfrak{A}, u \in \mathfrak{A} u \mathfrak{A}$. In particular, this holds if $\mathfrak{\Re}$ has an identity.

Proof. Let $k=k_{1}+k_{2}$ belong to $\Re$ where $k_{1} \in \mathfrak{A}$ and $k_{2} \in \mathfrak{U}^{\prime}$. Now $k_{1}$ $=\sum a_{i} k_{1} b_{i}$ where the $a_{i}$ and $b_{i}$ are in $\mathfrak{A}$. Hence $k_{1}=\sum a_{i} k b_{i} \in \Re$. Thus $k_{1} \in \Omega_{1}$. Since $\mathfrak{I}^{\prime}$ satisfies the same condition as $\mathfrak{A}$, a similar argument shows that $k_{2} \in \Omega_{2}$. Hence $\Re=\Omega_{1}+\Omega_{2}$. Since $\Omega_{1} \cap \Omega_{2}=\mathfrak{A} \cap \mathfrak{U}^{\prime}=0, \Omega=\Omega_{1} \oplus \Omega_{2}$.

4. Jordan homomorphisms of matrix rings and locally matrix rings. The key result which we shall obtain in this section is that any Jordan homo- 
morphism of a finite matrix ring is a sum of a homomorphism and an antihomomorphism.

Let $\mathfrak{U}$ be any ring that contains an identity 1 and a set of matrix units $e_{i j}, i, j=1,2, \cdots, n$, such that

$$
e_{i j} e_{k l}=\delta_{j k} e_{i l}, \quad \sum_{i=1}^{n} e_{i i}=1 .
$$

Let $\mathfrak{D}$ denote the subring of elements that commute with all the $\boldsymbol{e}_{i j}$. Then it is known $\left(^{3}\right)$ that every element of $\mathfrak{U}$ can be written in one and only one way in the form $\sum d_{i j} e_{i j}$, where $d_{i j} \in \mathfrak{D}$. Thus we may identify $\mathfrak{U}$ with the ring $\mathfrak{D}_{n}$ of $n \times n$ matrices with elements in $\mathfrak{D}$. We recall also that a necessary and sufficient condition that a ring with an identity be a matrix ring of the form $\mathfrak{D}_{n}$ is that it be decomposable as a direct sum of $n$ (operator) isomorphic right (left) ideals $\left({ }^{4}\right)$. It will be assumed throughout that $n \geqq 2$.

Let $J$ denote an arbitrary Jordan homomorphism of $\mathfrak{U}$ into a second ring. For $i \neq j$ define $e=e_{i i}+e_{i j}$. Then $e^{2}=e$ and, if $d \in \mathfrak{D}$, we have $[e, d]=0$. By Corollary 2 to Theorem 1 , it follows that $\left[e^{J}, d^{J}\right]=0$. Hence, $\left[e_{\mathfrak{t}}^{J}, d^{J}\right]$ $+\left[e_{i j}^{J}, d^{J}\right]=0$. Similarly $\left[e_{i i}^{J}, d^{J}\right]=0$, so that $\left[e_{i j}^{J}, d^{J}\right]=0$ and $d^{J}$ commutes with each $e_{i j}^{J}$. Again, by Corollary 2 to Theorem 1 , we have $\left(d e_{i i}\right)^{J}=d^{J} e_{i i}^{J}$. Furthermore, if $i \neq j$, then $\left(d e_{i j}\right)^{J}=\left(d e_{i j} e_{j j}+e_{j j} e_{i j} d\right)^{J}=d^{J} s_{i j}^{J} j_{j j}^{J}+e_{j j}^{J} e_{i j}^{J} d^{J}=d^{J}\left(e_{i j}^{J} j_{j j}^{J}+e_{j j}^{J} e_{i j}^{J}\right)$ $=d^{J} e_{i j}^{J}$. It follows that $\left(\sum d_{i j} e_{i j}\right)^{J}=\sum d_{i j}^{J} e_{i j}^{J}$. We thus obtain the following lemma.

\section{Lemma 2. The elements $d^{J}(d \in \mathfrak{D})$ and $e_{i j}^{J}$ commute and $\left(\sum d_{i j} e_{i j}\right)^{J}=\sum d_{i j}^{J} e_{i j}^{J}$.}

We now construct $\left(^{5}\right)$ two orthogonal $n \times n$ systems of matrix units in the enveloping ring $\mathfrak{E}$ of $\mathfrak{U}^{J}$. For arbitrary $i \neq j$, define the elements

$$
g_{i j}=\underset{e_{i i}}{J} \underset{i j}{J} e_{i j}^{J}, \quad h_{i j}=\underset{e_{i i}}{J} e_{j i}^{J} e_{j j}^{J}
$$

Since $e_{i j}=e_{i i} e_{i j} e_{j j}+e_{j j} e_{i j} e_{i i}$, we have

$$
\stackrel{J}{e_{i j}}=g_{i j}+h_{j i}
$$

Observe also that from the fact that $J$ preserves squares and from Corollary 2 to Theorem 1 we have $g_{i j} e_{j j}^{J}=e_{i i}^{J} g_{i j}=g_{i j}, g_{i j} e_{i i}^{J}=e_{j j}^{J} g_{i j}=0, h_{i j} e_{i l}^{J}=e_{j j}^{J} h_{i j}=0$ and $e_{i l}^{J} h_{i j}=h_{i j} e_{j j}^{J}=h_{i j}$. Hence it follows from (10) that

$$
g_{i j}=e_{i t}^{J} e_{i j}^{J}=e_{i j}^{J} e_{j j}^{J}, \quad h_{i j}=e_{i i}^{J} e_{j i}^{J}=e_{j i}^{J} e_{j j}^{J} \text {. }
$$

Moreover, if $i \neq j, j \neq k, k \neq l$, then $g_{i j} g_{k l}=0$. If $i \neq j, j \neq k$, and $i \neq k$, then $g_{i j} g_{j k}=g_{i j} e_{j j}^{J} e_{j k}^{J}=e_{i t}^{J} g_{i j} e_{j k}^{J}=e_{i i}^{J} e_{i j}^{J} e_{j k}^{J}$. But, since $e_{i i} e_{j k}=e_{j k} e_{i i}=0$, we have $e_{i t}^{J} e_{j k}^{J}=0$.

(3) $[6$, p. 57$]$.

(4) The sufficiency is a consequence of Theorem 5, p. 58 of [6]. The necessity is easy to establish.

(5) Cf. [11, pp. 147-149]. 
Therefore

$$
\begin{aligned}
g_{i j} g_{j k} & =e_{i i}^{J}\left(e_{i j}^{J} e_{j k}^{J}+e_{j k}^{J} e_{i j}^{J}\right) \\
& =e_{i i}^{J}\left(e_{i j} e_{j k}+e_{j k} e_{i j}\right)^{J} \\
& =e_{i i}^{J} e_{i k}^{J}=g_{i k} .
\end{aligned}
$$

Hence, for $i \neq j, k \neq l$, and $i \neq l, g_{i j} g_{k l}=\delta_{j k} g_{i l}$. Similarly $h_{i j} h_{k l}=\delta_{j k} h_{i l}$. Now, for each $i$, choose $j \neq i$ and define

$$
g_{i i}=g_{i j} g_{j i}, \quad h_{i i}=h_{i j} h_{j i} .
$$

Note first that $g_{i i}$ and $h_{i i}$ are independent of the choice of $j$. In fact, if $k \neq i$ and $k \neq j$, then $g_{i k} g_{k i}=g_{i k} g_{k j} g_{j i}=g_{i j} g_{j i}=g_{i i}$. Similarly $h_{i i}$ is independent of $j$. The elements $g_{i j}, h_{i j}$ are now defined for all $i, j$ and satisfy $g_{i j} g_{k l}=\delta_{j k} g_{i l}$, $h_{i j} h_{k l}=\delta_{j k} h_{i l}$ for all $i, j, k, l$. Observe that, for $i \neq j, e_{i j}^{J} e_{j l}^{J} e_{i l}^{J}=g_{i i}$ and $e_{j l}^{J} e_{i j}^{J} e_{i l}^{J}$ $=h_{i i}$. Addition of these equations leads to $e_{i 1}^{J}=g_{i i}+h_{i i}$. In other words, (10) also holds for $j=i$. It remains to prove $g_{i j} h_{k l}=0$. There is obviously no loss in assuming $i \neq j$ and $k \neq l$. We have $g_{i j} h_{k l}=e_{i j}^{J} e_{j j}^{J} e_{k k}^{J} e_{l k}^{J}$. If $k \neq j$, then $e_{j j}^{J} e_{k k}^{J}=0$ and hence $g_{i j} h_{k l}=0$. Therefore, assume $k=j$. Then $g_{i j} h_{j l}=g_{i j} h_{j i} h_{i l}=e_{i j}^{J} e_{j j}^{J} e_{i j}^{J} h_{i l}$ $=\left(e_{i j} e_{j j} e_{i j}\right)^{J} h_{i l}=0$. Similarly $h_{k l} g_{i j}=0$. This completes the proof of the next lemma.

LEMMA 3. The elements $g_{i j}$ and $h_{i j}$ constitute two $n \times n$ systems of matrix units in the enveloping ring $\mathbb{E}$ of $\mathfrak{U}^{J}$ such that $e_{\mathfrak{i}}^{J}=g_{i j}+h_{i j}$ and $g_{i j} h_{k l}=h_{k l} g_{i j}$ $=0$ for all $i, j, k, l$.

We are now in a position to prove the principal theorem of this section.

THEOREM 7. Any Jordan homomorphism $J$ of an $n \times n$ matrix ring $\mathfrak{U}=\mathfrak{D}_{n}$, $n \geqq 2$, is the sum of a homomorphism and an anti-homomorphism $\left(^{6}\right)$.

Proof. Let $g_{i j}, h_{i j}$ be the matrix units given in the above lemma and define $g=g_{11}+\cdots+g_{n n}, h=h_{11}+\cdots+h_{n n}$. Since $1=e_{11}^{J}+\cdots+e_{n n}^{J}$ and $e_{\mathfrak{H}}^{J}=g_{i i}+h_{i i}$, it follows that $1=g+h$. Obviously $g^{2}=g, h^{2}=h$, and $g h=h g=0$. Furthermore, $g$ and $h$ are in the center of the enveloping ring $\&$ of $\mathfrak{u}^{J}$. In fact, it is evident from Lemma 2 that all the $g_{i j}$ commute with the $d^{J}, d \in \mathfrak{D}$, and also $g e_{i j}^{J}=g_{i i} e_{i j}^{J}+g_{j j} e_{i j}^{J}=g_{i j}+g_{j j} h_{j i}=g_{i j}$. Similarly, $e_{i j}^{J} g=g_{i j}$ so that $g$ commutes with each $e_{i j}^{J}$. In the same way it is shown that $h$ commutes with each $e_{i j}^{J}$. But according to Lemma 2, $\&$ is generated by elements of the form $d^{J} e_{i j}^{J}, d \in \mathfrak{D}$; therefore, $g$ and $h$ are in the center of $\mathbb{E}$. It follows that $\mathbb{E}=\mathbb{E}_{g} \oplus \mathbb{E} h$, where

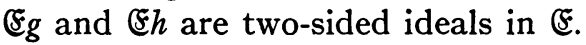

Define the mappings $J_{1}$ and $J_{2}$ of $\mathfrak{U}$ into $₹$ by $x^{J_{1}}=x^{J} g$ and $x^{J_{2}}=x^{J} h$ respectively. Evidently $J=J_{1}+J_{2}$. Now let $a, b$ be arbitrary elements of $\mathfrak{D}$. Then for $i \neq j$

$$
a b e_{i i}+b a e_{i j}=\left(a e_{i j}+b e_{j i}\right)^{2} .
$$

(6) This result implies Theorem 5 of [11] for the case $\mathfrak{A}$ not a division algebra. 
Therefore

$$
(a b)^{J} e_{i i}^{J}+(b a)^{J} e_{j i}^{J}=\left(a^{J} e_{i j}^{J}+b^{J} e_{j i}^{J}\right)^{2}=a^{J} b^{J} e_{i j}^{J} e_{j i}^{J}+b^{J} a^{J} e_{j i}^{J} e_{i j}^{J} .
$$

Multiplying on the right by $g_{i j}$, we obtain $(a b)^{J} g_{i j}=a^{J} b^{J} g_{i j}$. Similarly $(a b)^{J} h_{i j}$ $=b^{J} a^{J} h_{i j}$. If $x=\sum a_{i j} e_{i j}$ and $y=\sum b_{i j} e_{i j}$ are any two elements of $\mathfrak{u}$, then

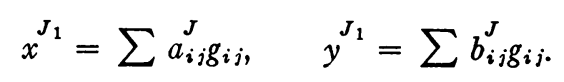

Therefore

$$
(x y)^{J_{1}}=\sum\left(a_{i k} b_{k j}\right)^{J} g_{i j}=\sum \underset{a_{i k}}{J} b_{k j}^{J} g_{i j}=x^{J_{1}} y^{J_{1}}
$$

and similarly $(x y)^{J_{2}}=y^{J_{2}} x^{J_{2}}$. Since $J_{1}$ and $J_{2}$ are obviously additive, it follows that $J_{1}$ is a homomorphism and $J_{2}$ is an anti-homomorphism of $\mathfrak{U}$.

COROLlARy. Let $\mathfrak{T}$ denote the ring of all linear transformations on a linear vector space $\mathfrak{X}$ over a division ring $\mathfrak{D}$, where the dimension of $\mathfrak{X}$ is at least two. Then any Jordan homomorphism of $\mathfrak{T}$ is the sum of a homomorphism and an anti-homomorphism.

Proof. The proof consists in showing that $\mathfrak{T}$ is a matrix ring for which we have only to exhibit a system of matrix units in $\mathfrak{T}$. Let $\left\{u_{\alpha}\right\}$ denote a basis for $\mathfrak{X}$. If $\mathfrak{X}$ is finite-dimensional, the result is well known; therefore, we can assume $\left\{u_{\alpha}\right\}$ to be infinite. Separate the elements of $\left\{u_{\alpha}\right\}$ into two disjoint sets $\left\{u_{\alpha}^{(1)}\right\},\left\{u_{\alpha}^{(2)}\right\}$ of equal power and define $E_{i j} \in \mathfrak{T}(i, j=1,2)$ by $u_{\alpha}^{(k)} E_{i j}$ $=\delta_{k i} u_{\alpha}^{(j)}$. Evidently these $E_{i j}$ constitute a $2 \times 2$ system of matrix units in $\mathfrak{T}$.

The method of proof in this corollary can be used to show that the ring of all bounded linear transformations in Hilbert space is a two-rowed matrix ring. We have only to take $\left\{u_{\alpha}\right\}$ as a complete orthonormal system in the Hilbert space.

We shall now make use of the results of the preceding section to extend our matrix theorem to the class of rings given in the following definition.

Definition. A ring $\mathfrak{B}$ is called locally matrix if any finite subset of $\mathfrak{B}$ can be embedded in a subring which is a matrix ring $\mathfrak{D}_{n}, n \geqq 2$.

An example of a locally matrix ring is a ring $\mathfrak{B}$ which possesses an infinite set $\left\{e_{\alpha \beta}\right\}$ of matrix units such that for arbitrary $x \in \mathfrak{B}$ there exists a finite set of indices $\alpha_{1}, \cdots, \alpha_{k}$ (depending on $x$ ) such that if $e=e_{\alpha_{1} \alpha_{1}}+\cdots$ $+e_{\alpha_{n} \alpha_{n}}$, then $x e=e x=x$. Another example of such a ring is an infinite direct (Kronecker) product of finite matrix algebras. Also, as we shall show in the next section, any simple ring which has minimal ideals is either locally matrix or a division ring.

THEOREM 8. Any Jordan homomorphism of a locally matrix ring is the sum of a homomorphism and an anti-homomorphism.

Proof. By Theorems 3 and 7 any Jordan homomorphism of a locally 
matrix ring $\mathfrak{B}$ is covered by the symmetrized direct sum mapping. We prove next that $\mathfrak{B}$ is the only ideal of this ring which contains all commutators. For this purpose let $x$ be any element of $\mathfrak{B}$ and let $\mathfrak{D}_{n}, n \geqq 2$, be a matrix subring containing $x$. Write $x=\sum a_{i j} e_{i j}$ where the $e_{i j}$ are the matrix units in $\mathfrak{D}_{n}$ and $a_{i j} \in \mathfrak{D}$. If $\mathfrak{J}$ is an ideal of $\mathfrak{B}$ containing all of the commutators, then $y$ contains $a_{i j} e_{i j}=\left[a_{i j} e_{i j}, e_{j j}\right]$ if $i \neq j$, and $J$ contains $a_{i i} e_{i i}=\left[a_{i i} e_{i j}, e_{j j}\right] e_{j i}$. Hence $x \in J$ and $J=\mathfrak{B}$. Observe next that $x=$ exe where $e=\sum e_{i i}$. Thus the conditions of Theorems 5 and 6 are satisfied. We can therefore apply the conclusions of these theorems and Theorem 4 to obtain the present result.

5. Jordan homomorphisms of primitive rings with minimal ideals. We recall that a ring $\mathfrak{B}$ is defined to be primitive $\left({ }^{7}\right)$ if it contains a maximal right ideal $\Re$ such that the quotient $(\Re: \mathfrak{B})=(0)$. This is equivalent to the condition that $\mathfrak{B}$ is isomorphic to an irreducible ring of endomorphisms. If $\mathfrak{B}$ is primitive and contains minimal ideals, then the structure of $\mathfrak{B}$ can be described more precisely as follows. One can associate with $\mathfrak{B}$ a pair of dual vector spaces $\mathfrak{X}, \mathfrak{X}^{\prime}$. Here $\mathfrak{X}$ is a left vector space over a division ring $\Delta, \mathfrak{X}^{\prime}$ is a right vector space over $\Delta$, and there is defined a bilinear form $\left(x, y^{\prime}\right), x$ in $\mathfrak{X}, y^{\prime}$ in $\mathfrak{X}^{\prime}$, which is nondegenerate in the sense that $\left(z, y^{\prime}\right)=0$ for all $y^{\prime}$ implies $z=0$, and $\left(x, z^{\prime}\right)=0$ for all $x$ implies $z^{\prime}=0$. The ring $\mathfrak{B}$ can be regarded as a ring of linear transformations in $\mathfrak{X}$ which have adjoints relative to $\left(x, y^{\prime}\right)$. Also $\mathfrak{B}$ contains all transformations of the form

$$
x \rightarrow\left(x, u_{1}^{\prime}\right) v_{1}+\left(x, u_{2}^{\prime}\right) v_{2}+\cdots+\left(x, u_{r}^{\prime}\right) v_{r}
$$

where the $u_{i}^{\prime} \in \mathfrak{X}^{\prime}$ and the $v_{i} \in \mathfrak{X}$. These elements are sums of elements of rank one: $x \rightarrow\left(x, u^{\prime}\right) v, u^{\prime}, v \neq 0$. We shall find it convenient to denote the mapping $x \rightarrow\left(x, u^{\prime}\right) v$ by $u^{\prime} \times v$. Then it is easy to see that the function $u^{\prime} \times v$ is additive in $u^{\prime}$ and in $v$ and that $u^{\prime} \alpha \times v=u^{\prime} \times \alpha v$ for $\alpha \in \Delta$. Also we have the multiplication rule.

$$
\left(u_{1}^{\prime} \times v_{1}\right)\left(u_{2}^{\prime} \times v_{2}\right)=u_{1}^{\prime}\left(v_{1}, u_{2}^{\prime}\right) \times v_{2} .
$$

The elements of the form (11) constitute a two-sided ideal $\mathfrak{F}$ of $\mathfrak{B}$ which is contained in every nonzero two-sided ideal. The sub-ring $\mathfrak{F}$ of $\mathfrak{B}$ is a simple ring with minimal ideals. Moreover, as has been shown by Dieudonné [4], any simple ring with minimal ideals is of this type. The centralizer of $\mathfrak{F}$ in $\mathfrak{B}$ is equal to the center of $\mathfrak{B}$ which is a commutative integral domain. If the dimension of $\mathfrak{X}$ over $\Delta$ is infinite, then the center of $\mathfrak{F}$ is necessarily zero. If $a$ is any nonzero element of $\mathfrak{B}$, then there exists $f \in \mathfrak{F}$ such that faf $\neq 0$. In fact, let $v$ be an element of $\mathfrak{X}$ such that $v a \neq 0$ and choose $u^{\prime} \in \mathfrak{X}^{\prime}$ such that $\left(v a, u^{\prime}\right)$ $\neq 0$. Then clearly $\left(u^{\prime} \times v\right) a\left(u^{\prime} \times v\right) \neq 0$.

Lemma 4. Let $\mathfrak{S}$ and $\mathfrak{S}^{\prime}$ be finite-dimensional subspaces of $\mathfrak{X}$ and $\mathfrak{X}^{\prime}$ respectively. Then these spaces can be embedded in finite-dimensional subspaces $\mathfrak{U}$ and

(7) Nearly all the definitions and results assumed in this section can be found in [8]. 
$\mathfrak{u}^{\prime}$ respectively which are dual relative to the given bilinear form.

Proof. Let $x_{1}^{\prime} ;, \cdots, x_{r}^{\prime}$ be a basis for $\mathfrak{S}^{\prime}$ and choose elements $x_{1}, \cdots, x_{r}$ $\in \mathbb{X}$ such that $\left(x_{i}, x_{j}^{\prime}\right)=\delta_{i j}$ for $\left.i, j=1, \cdots, r{ }^{8}\right)$. Consider the subspace $\mathfrak{U}=\mathfrak{S}+\left[x_{1}, \cdots, x_{r}\right]$ and observe that $r \leqq \operatorname{dim} \mathfrak{u}$. If $r<\operatorname{dim} \mathfrak{u}$, then there exists $x_{r+1} \neq 0 \in \mathfrak{U}$ such that $\left(x_{r+1}, x_{i}^{\prime}\right)=0$ for $i=1, \cdots, r$. Evidently $x_{1}, \cdots$, $x_{r+1}$ are linearly independent so that there exists $x_{r+1}^{\prime} \in \mathfrak{X}^{\prime}$ such that $\left(x_{r+1}\right.$, $\left.x_{r+1}^{\prime}\right)=1$ and $\left(x_{i}, x_{r+1}^{\prime}\right)=0$ for $i=1, \cdots, r$. Repeating this process, we finally obtain a basis $x_{1}, \cdots, x_{8}$ for $\mathfrak{U}$ and elements $x_{1}^{\prime}, \cdots, x_{s}^{\prime} \in \mathfrak{X}$ such that $\left(x_{i}, x_{j}^{\prime}\right)=\delta_{i j}$ for $i, j=1, \cdots, s$. The subspaces $\mathfrak{U}$ and $\mathfrak{u}^{\prime}=\left[x_{1}^{\prime}, \cdots, x_{s}^{\prime}\right]$ obviously satisfy the desired conditions.

We can now prove the following result which is due to Litoff (unpublished).

THEOREM 9. If $\mathfrak{F}$ is a simple ring with minimal ideals, then any finite subset of $\mathfrak{F}$ can be embedded in a subring of $\mathfrak{F}$ which is isomorphic to $\Delta_{n}$, where $\Delta$ is the division ring associated with $\mathfrak{F}$.

Proof. Since any element of $\mathfrak{F}$ is a sum of elements of rank one, it suffices to take the finite set to be $u_{1}^{\prime} \times v_{1}, u_{2}^{\prime} \times v_{2}, \cdots, u_{m}^{\prime} \times v_{m}$. Let $\varsigma^{\prime}$ $=\left[u_{1}^{\prime}, \cdots, u_{m}^{\prime}\right]$ and $\subseteq=\left[v_{1}, \cdots, v_{m}\right]$ and embed these subspaces respectively in finite-dimensional dual subspaces $\mathfrak{u}^{\prime}$ and $\mathfrak{u}$. Choose bases $\left(x_{1}^{\prime}, \cdots, x_{n}^{\prime}\right)$ and $\left(x_{1}, \cdots, x_{n}\right)$ for $\mathfrak{U}^{\prime}$ and $\mathfrak{U}$ such that $\left(x_{i}, x_{j}^{\prime}\right)=\delta_{i j}$. Then the elements $e_{i j}=x_{i}^{\prime} \times x_{j}$ are matrix units and the subring $\mathfrak{M}$ of $\mathfrak{F}$ consisting of elements of the form $\sum x_{i}^{\prime} \alpha_{i j} \times x_{j}, \alpha_{i j} \in \Delta$, is a matrix ring isomorphic to $\Delta_{n}$. Since $u_{k}^{\prime}=\sum x_{i}^{\prime} \lambda_{i}$ and $v_{k}=\sum \mu_{j} x_{j}$, we have $u_{k}^{\prime} \times v_{k}=\sum x_{i}^{\prime} \lambda_{i} \mu_{j} \times x_{j} \in \mathfrak{M}$.

This result implies that any simple ring with minimal ideals is either a division ring or a locally matrix ring. Hence we have the following theorem.

THEOREM 10. Any Jordan homomorphism of a simple ring with minimal ideals which is not a division ring is a sum of a homomorphism and an antihomomorphism.

Let $\mathfrak{A}$ be any indecomposable ring which has the property that any Jordan homomorphism of $\mathfrak{A}$ is a sum of a homomorphism and an anti-homomorphism. By indecomposability we mean that if $\mathfrak{A}=\mathfrak{A}_{1} \oplus \mathfrak{A}_{2}$, where $\mathfrak{A}_{1}$ and $\mathfrak{A}_{2}$ are ideals in $\mathfrak{A}$, then either $\mathfrak{A}_{1}=(0)$ or $\mathfrak{A}_{2}=(0)$. Let $J$ be a Jordan homomorphism of $\mathfrak{A}$ onto itself. Then it follows directly that $J$ is either a homomorphism or antihomomorphism. Thus we see that any Jordan homomorphism of an indecomposable locally matrix ring onto itself, and hence of any simple ring with minimal ideals (Theorems 2, 10), is either a homomorphism or an antihomomorphism $\left({ }^{9}\right)$. We proceed to show that this property can be carried

(8) Cf. [7, Lemma 1].

( ${ }^{\circ}$ This contains the result recently announced by Hua [5] that any Jordan automorphism of a simple ring which satisfies the descending chain condition is either an automorphism or an anti-automorphism. 
over to general primitive rings with minimal ideals. This result is also a consequence of a more general theorem which we shall obtain later (Theorem 21). However, the present method is also of interest since it can be applied in other cases.

LeMma 5. Let $\mathfrak{B}$ be a ring which contains a nonzero ideal $\mathfrak{F}$ such that the following conditions hold: (i) the centralizer of $\mathfrak{F}$ is equal to the center $\mathfrak{C}$ of $\mathfrak{B}$, (ii) $\mathfrak{C} \cap \mathfrak{F}=(0)$, (iii) no nonzero element of $\mathfrak{E}$ is a zero divisor. Let $J$ be a Jordan homomorphism of $\mathfrak{B}$ onto itself which induces a homomorphism (anti-homomorphism) of $\mathfrak{F}$ onto itself. Then $J$ is a homomorphism (anti-homomorphism).

Proof. Let $f, g \in \mathfrak{F}$ and $a, b \in \mathfrak{B}$. Then $(a f)^{J}-a^{J} f^{J}=f^{J} a^{J}-(f a)^{J}, g^{J}\left((a f)^{J}\right.$ $\left.-a^{J} f^{J}\right)=(g a f)^{J}-g^{J} a^{J} f^{J}$ and $\left(f^{J} a^{J}-(f a)^{J}\right) g^{J}=f^{J} a^{J} g^{J}-(f a g)^{J}$. Since $(g a f)^{J}$ $-g^{J} a^{J} f^{J}=f^{J} a^{J} g^{J}-(f a g)^{J}$, it follows that $(a f)^{J}-a^{J} f^{J}$ commutes with every $g^{J}$. By (i), (af) $)^{J}-a^{J} f^{J}$ is in the center and, by (ii), $(a f)^{J}=a^{J} f^{J}$. Since $J$ is a Jordan homomorphism, we have also that $(f a)^{J}=f^{J} a^{J}$. By an argument similar to the foregoing, we can now prove that $(a b)^{J}-a^{J} b^{J}$ commutes with every $f^{J}$. Hence $(a b)^{J}-a^{J} b^{J}$ is in $\mathfrak{c}$. Since $\left[(a b)^{J}-a^{J} b^{J}\right]\left[(a b)^{J}-b^{J} a^{J}\right]=0$, it follows from (iii) that either $(a b)^{J}=a^{J} b^{J}$ or $(a b)^{J}=b^{J} a^{J}$. Hence by Hua's lemma, $J$ is either a homomorphism or an anti-homomorphism in $\mathfrak{B}$. Since $\mathfrak{F}$ is not commutative (by (i) and (ii)) and since $J$ maps $\mathfrak{F}$ homomorphically, $J$ maps $\mathfrak{B}$ homomorphically. A similar argument applies to the case in which $J$ is an anti-homomorphism.

In order to apply this result to primitive rings with minimal ideals, we require some information concerning the ideal structure of the associated special Jordan rings.

A subset $\mathfrak{J}$ of a ring $\mathfrak{A}$ is called a Jordan ideal in $\mathfrak{A}$ provided (1) $\mathfrak{I}$ is a group under addition, (2) J contains $\{a z\}=a z+z a$ for all $a \in \mathfrak{A}$ and $z \in \mathfrak{J}$, (3) $\mathfrak{J}$ contains the elements $z^{2}, a z a, z a z$ for all $a \in \mathfrak{A}$ and $z \in \mathcal{J}$. If $\mathfrak{J}$ admits the operator $1 / 2$, then (1) and (2) imply (3). This is a consequence of the relations $2 u^{2}=\{u u\}$ and $2 u v u=\{u\{v u\}\}-\left\{v u^{2}\right\}$. From the relations uvw $+w v u=(u+w) v(u+w)-u v u-w v w$ and $u v w+w v u=\{u\{v w\}\}-(u w v+v w u)$ $=\{w\{v u\}\}-(w u v+v u w)$, it follows that elements of the form $u v w+w v u$ are in $J$ provided either $u, v$, or $w$ is in $J$. Under the above definition, the kernel of a Jordan homomorphism of $\mathfrak{A}$ is a Jordan ideal in $\mathfrak{A}\left({ }^{10}\right)$.

THEOREM 11. Every Jordan ideal in a locally matrix ring is an ordinary ideal.

Proof. Let $\mathfrak{J}$ be a Jordan ideal in a locally matrix ring $\mathfrak{A}$. We have only to prove that $a z$ and $z a$ belong to $\mathfrak{J}$ for all $a \in \mathfrak{A}$ and $z \in \mathfrak{J}$. Let $\mathfrak{D}_{n}(n \geqq 2)$ denote a matrix subring of $\mathfrak{A}$ which contains the elements $a, z$ and observe that $\mathfrak{J} \cap \mathfrak{D}_{n}$

(10) It is not known whether or not the homomorphic image of a special Jordan ring is a special Jordan ring. This is an unsolved problem even for the case of finite-dimensional algebras. 
is a Jordan ideal in $\mathfrak{D}_{n}$. Thus it is sufficient to prove the theorem for the case in which $\mathfrak{A}$ is a matrix ring $\mathfrak{D}_{n}(n \geqq 2)$.

Let $\left\{e_{i j}\right\}$ be the matrix units in $\mathfrak{D}_{n}$ and denote by $\Lambda$ the collection of all $\alpha \in \mathfrak{D}$ such that $\alpha e_{i j} \in J$ for some $i, j$. Note that, if $\alpha e_{i i} \in J$, then $\alpha e_{i j}=\left\{\alpha e_{i i}, e_{i j}\right\}$ for $i \neq j$ so that $\Lambda$ consists of all $\alpha \in \mathfrak{D}$ such that $\alpha e_{i j} \in J$ for some $i \neq j$. We prove that $\Lambda_{n}$ is an ideal in $\mathfrak{D}_{n}$ and that $\Lambda_{n}=\mathfrak{J}$.

Let $\alpha$ be an arbitrary element of $\Lambda$ and choose $i \neq j$ such that $\alpha e_{i j} \in \mathcal{J}$. For every $p$ we have $\alpha e_{p j}=e_{p i}\left(\alpha e_{i j}\right) e_{j j}+e_{j j}\left(\alpha e_{i j}\right) e_{p i}$. Therefore $\alpha e_{p j} \in J$. Now consider arbitrary $p, q$. If $p=q=j$, then we already have $\alpha e_{p q} \in J$ J. Hence assume either $p \neq j$ or $q \neq j$. Then $\alpha e_{p q}=e_{p p}\left(\alpha e_{p j}\right) e_{j q}+e_{j q}\left(\alpha e_{p j}\right) e_{p p}$. Therefore $\alpha e_{p q} \in J$ for all $p, q$ and consequently $\Lambda_{n} \subseteq$ J. It also follows here that $\Lambda$ is a group under addition. Furthermore let $\alpha \in \Lambda, d \in \mathfrak{D}$ and choose $i \neq j$ such that $\alpha e_{i j} \in J$. Since $\alpha d e_{i j}=\left\{\alpha e_{i j}, d e_{j j}\right\}$ and $d \alpha e_{i j}=\left\{d e_{i i}, \alpha e_{i j}\right\}$, we have $\alpha d \in \Lambda$ and $d \alpha \in \Lambda$. Therefore $\Lambda$ is an ideal in $\mathfrak{D}$ so that $\Lambda_{n}$ is an ideal in $\mathfrak{D}_{n}$. Next let $a=\sum \alpha_{i j} e_{i j}$ be an arbitrary element of $\mathfrak{J}$. Then $\alpha_{i j} e_{i j}=e_{i j}\left(e_{j i} a e_{j i}\right) e_{i j}$ so that $\alpha_{i j} e_{i j} \in J$ for all $i, j$. This implies $\alpha_{i j} \in \Lambda$ and hence $a \in \Lambda_{n}$. In other words $J \subseteq \Lambda_{n}$. Therefore $J=\Lambda_{n}$.

Now let $\mathfrak{B}$ denote a primitive ring with minimal ideals and let $\mathfrak{F}$ be the minimal two-sided ideal contained in $\mathfrak{B}$. We assume that $\mathfrak{B}$ is not a division ring, in which case $\mathfrak{F}$ is a locally matrix ring.

THEOREM 12. Every nonzero Jordan ideal in $\mathfrak{B}$ contains $\mathfrak{F}$.

Proof. Let $\mathfrak{J}$ be a nonzero Jordan ideal in $\mathfrak{B}$ and set $\mathfrak{J}_{0}=\mathfrak{J} \cap \mathfrak{F}$. Clearly $\mathfrak{J}_{0}$ is a Jordan ideal in $\mathfrak{F}$. Furthermore, if $a$ is a nonzero element of $\mathfrak{B}$, then there exists an element of $f \in \mathfrak{F}$ such that $f a f \neq 0$. Since $f a f \in J_{0}$, it follows that $J_{0} \neq(0)$. By Theorem $11, J_{0}$ is an ideal in $\mathfrak{F}$ so that $J_{0}=\mathfrak{F}$, since $\mathfrak{F}$ is simple. In other words $\mathfrak{F} \subseteq$ J

TheOREM 13. Any Jordan homomorphism of $\mathfrak{B}$ onto itself which does not map $\mathfrak{F}$ into zero is either an isomorphism or anti-isomorphism.

Proof. Denote the Jordan homomorphism by $J$ and observe that the kernel of the homomorphism is a Jordan ideal in $\mathfrak{B}$. By Theorem 12 the kernel must either be zero or contain $\mathfrak{F}$, and the latter possibility is ruled out since $\mathfrak{F}$ is not mapped into zero. It follows that $J$ is a Jordan isomorphism of $\mathfrak{B}$ onto itself. Furthermore $\mathfrak{F}^{J}$ is a nonzero Jordan ideal in $\mathfrak{B}$ so that $\mathfrak{F} \subseteq \mathfrak{F}^{J}$. A similar argument using the inverse mapping $J^{-1}$ gives $\mathfrak{F}^{J} \subseteq \mathfrak{F}$ and hence $\mathfrak{F}^{J}=\mathfrak{F}$. Therefore $J$ is a Jordan isomorphism of $\mathfrak{F}$ onto itself. Since $\mathfrak{F}$ is a simple locally matrix ring, it follows from Theorem 8 and the remarks preceding Lemma 5 that $J$ is either an isomorphism or anti-isomorphism of $\mathfrak{F}$ onto $\mathfrak{F}$. An application of Lemma 5 now completes the proof.

6. Lie triple system homomorphisms and Jordan homomorphisms. In this section we develop a Lie ring method for the study of Jordan homomorphisms. If $J$ is a Jordan homomorphism of a ring $\mathfrak{A}$ into a ring $\mathfrak{B}$ and is at the same time a Lie homomorphism in the sense that $[a b]^{J}=\left[a^{J} b^{J}\right]$, then $2(a b)^{J}=(\{a b\}+[a b])^{J}=\left(\left\{a^{J} b^{J}\right\}+\left[a^{J} b^{J}\right]\right)=2 a^{J} b^{J}$. Hence if $\mathfrak{B}$ has no ele- 
ments of additive order 2 , then $J$ is a homomorphism. Similarly if $J$ is a Jordan homomorphism and a Lie anti-homomorphism in the sense that $[a b]^{J}=\left[b^{J} a^{J}\right]$ and $\mathfrak{B}$ has no elements of additive order 2 , then $J$ is an antihomomorphism. Now we know that any Jordan homomorphism is a Lie triple system homomorphism, that is, $[[a b] c]^{J}=\left[\left[a^{J} b^{J}\right] c^{J}\right]$. Hence, we raise the question: Under what conditions is a Lie triple system homomorphism of a ring either a Lie homomorphism or a Lie anti-homomorphism?

More generally let $\mathfrak{R}$ be an arbitrary special Lie ring and let $T$ be a Lie triple system homomorphism of $\mathfrak{R}$. The image $\mathfrak{R}^{T}$ is a Lie triple system and from this it follows easily that the enveloping Lie ring $\mathfrak{M}$ of $\mathbb{R}^{T}$ is the totality $\mathfrak{R}^{T}+\left[\mathfrak{R}^{T} \mathfrak{R}^{T}\right]$ of elements of the form $a^{T}+\sum\left[b_{i}^{T} c_{i}^{T}\right], a, b_{i}, c_{i}$ in $\mathfrak{R}\left({ }^{11}\right)$.

We now define $\mathfrak{M}^{+}$and $\mathfrak{M}^{-}$to be the sets of all finite sums of elements of the form $[a b]^{T}-\left[a^{T} b^{T}\right]$ and $[a b]^{T}-\left[b^{T} a^{T}\right]$ respectively. Observe that $\mathfrak{M}^{+}$ $=(0)$ is necessary and sufficient for $T$ to be a Lie homomorphism of $\mathbb{R}$ and $\mathfrak{M}^{-}=(0)$ is necessary and sufficient for $T$ to be a Lie anti-homomorphism. We prove now the following result.

THEOREM 14. $\mathfrak{M}^{+}$and $\mathfrak{M}^{-}$are Lie ideals $\left.{ }^{12}\right)$ in $\mathfrak{M}$ and $\left[\mathfrak{M}^{+}, \mathfrak{M}^{-}\right] \subseteq \subseteq$ the center of $\mathfrak{M}$.

Proof. It is obvious that $\mathfrak{M}^{+}$and $\mathfrak{M}^{-}$are additive groups. Furthermore,

$$
\left[[a b]^{T}-\left[a^{T} b^{T}\right], c^{T}\right]=\left[[a b]^{T} c^{T}\right]-\left[\left[a^{T} b^{T}\right] c^{T}\right]=\left[[a b]^{T} c^{T}\right]-[[a b] c]^{T} .
$$

Therefore $\mathfrak{M}^{+}$is a Lie ideal. Similarly $\mathfrak{M}^{-}$is a Lie ideal in $\mathfrak{M}$. Also,

$$
\begin{aligned}
& {\left[\left[[a b]^{T}-\left[a^{T} b^{T}\right],[c d]^{T}-\left[d^{T} c^{T}\right]\right] x^{T}\right] } \\
&= {\left[\left[[a b]^{T}[c d]^{T}\right] x^{T}\right]-\left[\left[[a b]^{T}\left[d^{T} c^{T}\right]\right] x^{T}\right] } \\
&-\left[\left[\left[a^{T} b^{T}\right][c d]^{T}\right] x^{T}\right]+\left[\left[\left[a^{T} b^{T}\right]\left[d^{T} c^{T}\right]\right] x^{T}\right] \\
&= {[[[a b][c d]] x]^{T}-\left[[[a b][d c]]^{T} x^{T}\right] } \\
&-\left[[[a b][c d]]^{T} x^{T}\right]-\left[\left[\left[a^{T} b^{T}\right]\left[c^{T} d^{T}\right]\right] x^{T}\right] \\
&= {[[[a b][c d]] x]^{T}-\left[\left[\left[a^{T} b^{T}\right]\left[c^{T} d^{T}\right]\right] x^{T}\right] . }
\end{aligned}
$$

By the Jacobi identity, $\left[\left[\left[a^{T} b^{T}\right]\left[c^{T} d^{T}\right]\right] x^{T}\right]=\left[\left[a^{T} b^{T}\right]\left[\left[c^{T} d^{T}\right] x^{T}\right]\right]+\left[\left[\left[a^{T} b^{T}\right]\right.\right.$ $\left.\left.x^{T}\right]\left[c^{T} d^{T}\right]\right]=\left[[a b][[c d] x]^{T}+[[[a b] x][c d]]^{T}=[[[a b][c d]] x]^{T}\right.$. Therefore it follows that $\left[\mathfrak{M}^{+}, \mathfrak{M}^{-}\right] \subseteq \mathfrak{S}$.

TheOREM 15. Let $T$ be a Lie triple system homomorphism of the special Lie ring $\mathfrak{R}$ and denote by $\mathfrak{M}$ the enveloping Lie ring of $\mathfrak{R}^{T}$ and $\mathbb{S}$ the center of $\mathfrak{M}$. Assume (i) $\mathfrak{M} / \mathbb{C}$ has no commutative Lie ideals and (ii) any two nonzero Lie ideals in $\mathfrak{M} / \mathfrak{S}$ have a nonzero intersection. Then $T$, when restricted to the Lie

(ii) Cf. [10, p. 155].

(12) An ideal in a special Lie ring is defined to be a subgroup of the additive group which is closed under commutation with arbitrary elements of the Lie ring. 
ring $[\mathbb{R}, \mathbb{R}]$, is either a Lie homomorphism or anti-homomorphism.

Proof. Using the notation in Theorem 14 , we set $\mathfrak{U}=\left(\mathfrak{M}^{+}+\mathfrak{E}\right) \cap\left(\mathfrak{M}^{-}+\mathfrak{S}\right)$ and observe that $[\mathfrak{u}, \mathfrak{u}] \subseteq \mathfrak{E}$. Therefore $[\mathfrak{u} / \mathfrak{C}, \mathfrak{u} / \mathfrak{E}]=(0)$ and since $\mathfrak{u} / \mathfrak{S}$ is a Lie ideal in $\mathfrak{M} / \mathfrak{S}$ it follows by condition (i) that $\mathfrak{U} / \mathfrak{S}=(0)$. Hence $\mathfrak{U}=\mathfrak{S}$ so that $\left(\left(\mathfrak{M}^{+}+\mathfrak{S}\right) / \mathfrak{S}\right) \cap((\mathfrak{M}-+\mathfrak{S}) / \mathfrak{S})=(0)$. By condition (ii) this implies either $\mathfrak{M}^{+} \subseteq \mathbb{E}$ or $\mathfrak{M}^{-} \subseteq \mathfrak{E}$. If $\mathfrak{M}^{+} \subseteq \mathbb{E}$, then

$$
\left[[a b]^{T} c^{T}\right]-[[a b] c]^{T}=\left[[a b]^{T}-\left[a^{T} b^{T}\right], c^{T}\right]=0
$$

for all $a, b, c$. In particular, $[[a b][c d]]^{T}=\left[[a b]^{T}[c d]^{T}\right]$. In other words, $T$ is a Lie homomorphism on $[\mathbb{R}, \mathbb{R}]$. Similarly, if $\mathfrak{M}-\subseteq \mathbb{E}$ then $T$ is a Lie anti-homomorphism on $[R, R]$.

COROLlaRy. Let $\mathfrak{R}$ be a simple Lie ring in which $[\mathbb{R}, \mathbb{R}] \neq(0)$. Then any Lie triple system homomorphism of $\mathfrak{R}$ onto itself is either a Lie homomorphism or anti-homomorphism.

Since a Jordan homormorphism of a ring is also a Lie triple system homomorphism, the above results apply to this case. Moreover, by exploiting the special properties of a Jordan homomorphism we obtain the following theorem.

THEOREM 16. Let $J$ be a Jordan homomorphism of a ring $\mathfrak{A}$ into a ring $\mathfrak{B}$ and denote by $\mathfrak{M}$ the enveloping Lie ring of $\mathfrak{A}^{J}$ and $\mathbb{E}$ the center of $\mathfrak{M}$. Assume (i) $\mathfrak{M} / \mathfrak{C}$ has no commutative Lie ideals, (ii) any two nonzero Lie ideals in $\mathfrak{M} / \mathfrak{S}$ have a nonzero intersection, (iii) no nonzero element of $\mathfrak{S}$ is a zero divisor in $\mathfrak{M}$ and (iv) $\mathfrak{B}$ contains no elements of additive order two. Then $J$ is either a homomorphism or anti-homomorphism.

Proof. As in the proof of Theorem 11 either $\mathfrak{M}^{+} \subseteq \mathfrak{S}$ or $\mathfrak{M}^{-} \subseteq \subseteq \mathfrak{S}$ where $\mathfrak{M}^{+}$ and $\mathfrak{M}^{-}$are defined as before with $T=J$. Furthermore,

$$
\begin{aligned}
{[a b]^{J}-\left[a^{J} b^{J}\right] } & =(a b)^{J}-(b a)^{J}-a^{J} b^{J}+b^{J} a^{J} \\
& =(a b)^{J}-(b a)^{J}-2 a^{J} b^{J}+a^{J} b^{J}+b^{J} a^{J} \\
& =2\left[(a b)^{J}-a^{J} b^{J}\right] .
\end{aligned}
$$

Similarly $[a b]^{J}-\left[b^{J} a^{J}\right]=2\left[(a b)^{J}-b^{J} a^{J}\right]$. Hence, by relation $(7)$ in $\S 1$,

$$
\left([a b]^{J}-\left[a^{J} b^{J}\right]\right)\left([a b]^{J}-\left[b^{J} a^{J}\right]\right)=0 .
$$

It follows by condition (iii) that either $[a, b]^{J}=\left[a^{J} b^{J}\right]$ or $[a b]^{J}=\left[b^{J} a^{J}\right]$ for all $a, b$. Therefore, by Lemma $1, J$ must be either a Lie homomorphism or anti-homomorphism. The desired result now follows from the remarks at the beginning of this section.

7. Further properties of Jordan homomorphisms. In Theorem 16 of the preceding section we have obtained sufficient conditions under which a Jordan homomorphism $J$ of one ring $\mathfrak{A}$ into a second ring $\mathfrak{B}$ reduces to either a homo- 
morphism or anti-homomorphism. The proof was based on results obtained for general Lie triple system homomorphisms. By exploiting from the outset the fact that $J$ is a Jordan homomorphism, we obtain in this section a theorem similar to Theorem 16 but without any restriction on the additive order of elements of $\mathfrak{B}$. This is accomplished by considering in place of the enveloping Lie ring of $\mathfrak{U}^{J}$ another, in general larger, Lie ring.

Let $\Im$ be any special Jordan ring and denote its enveloping Lie ring by $\mathfrak{M}$. Consider the set $\mathfrak{R}$ of all finite sums of the form $x+\sum y_{i} z_{i}$ where $x, y_{i}, z_{i}$ are elements of $\Im$. It is obvious that $\mathfrak{M} \subseteq \mathfrak{N}$ and since $2 y z=\{y z\}+[y z]$, we have $\mathfrak{M}=\mathfrak{N}$ in case $\mathfrak{M}$ admits the operator $1 / 2$. From the obvious identities

$$
\begin{aligned}
{[x, y z] } & =(x y z+z y x)-(y z+z y) x, \\
{[x y, u v] } & =x(y u v+v u y)-(u v x+x v u) y
\end{aligned}
$$

and the fact that expressions of the form $x y z+z y x, y z+z y$ are Jordan polynomials, it follows easily that $\mathfrak{R}$ is a Lie ring. We shall call $\mathfrak{N}$ the extended enveloping Lie ring of $\Im$. The following example shows that $\mathfrak{N}$ is, in general, actually larger than $\mathfrak{M}$. Let $\Re$ be any noncommutative ring every element of which has additive order two. Consider the symmetrized direct sum $\Re \oplus \Re^{\prime}$ and let $\Im$ be the special Jordan subring of $\Re \oplus \Re^{\prime}$ consisting of all elements of the form $\bar{r}=r+r^{\prime}$. Observe that $\overline{x y}-\bar{y} \bar{x}=[x y]$ is an element of $\mathfrak{N}$ while every element of $\mathfrak{M}$ has the form

$$
\bar{a}+\sum\left[\bar{b}_{i} \bar{c}_{i}\right]=a+\sum\left[b_{i} c_{i}\right]+\left(a-\sum\left[b_{i} c_{i}\right]\right)^{\prime} .
$$

Hence if $\overline{x y}-\bar{y} \bar{x}$ were in $\mathfrak{M}$, then there would exist $a, b_{i}, c_{i}$ such that

$$
a+\sum\left[b_{i} c_{i}\right]=[x y], \quad a-\sum\left[b_{i} c_{i}\right]=0 .
$$

Adding these relations we obtain $[x y]=2 a=0$. Therefore if $[x y] \neq 0$, then $\overline{x y}-\bar{y} \bar{x} \notin \mathfrak{M}$.

We return now to the Jordan mapping $J$ of $\mathfrak{A}$ into $\mathfrak{B}$ and denote by $\mathfrak{N}$ the extended enveloping Lie ring of $\mathfrak{A}^{J}$. The identities (13), (14) become

$$
\begin{aligned}
{\left[a^{J}, b^{J} c^{J}\right] } & =(a b c+c b a)^{J}-(b c+c b)^{J} a^{J}, \\
{\left[a^{J} b^{J}, c^{J} d^{J}\right] } & =a^{J}(b c d+d c b)^{J}-(a d c+c d a)^{J} b^{J} .
\end{aligned}
$$

Two successive applications of (15) lead to

$$
\begin{aligned}
{\left[\left[a^{J}, b^{J} c^{J}\right] d^{J}\right]=} & (a c b d+d b c a-c b a d-d a b c)^{J} \\
& +(a b c+c b a-a c b-b c a)^{J} d^{J}
\end{aligned}
$$

and an application of (16) followed by two applications of (15) lead to

$$
\begin{aligned}
{\left[\left[a^{J} b^{J}, c^{J} d^{J}\right] x^{J}\right]=} & (b a d c x+x c d a b-d c b a x-x a b c d)^{J} \\
& +(a b c d+d c b a-b a d c-c d a b)^{J} x^{J}
\end{aligned}
$$

Next define the sets $\mathfrak{N}^{+}$and $\mathfrak{N}^{-}$to consist of all finite sums of elements 
of the form $(a b)^{J}-a^{J} b^{J}$ and $(a b)^{J}-b^{J} a^{J}$ respectively. The analogue of Theorem 14 holds for $\mathfrak{N}^{+}$and $\mathfrak{N}^{-}$.

TheoRem 17. $\mathfrak{N}^{+}$and $\mathfrak{N}^{-}$are Lie ideals in $\mathfrak{N}$ and $\left[\mathfrak{N}^{+}, \mathfrak{N}^{-}\right] \subseteq \subseteq$, the center

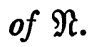

Proof. It is obvious that $\mathfrak{R}^{+}$and $\mathfrak{N}^{-}$are additive groups. Furthermore, by identity (15) above,

$$
\left[(a b)^{J}-a^{J} b^{J}, c^{J}\right]=(c a b)^{J}-c^{J}(a b)^{J}+(b a c)^{J}-(b a)^{J} c^{J},
$$

and, by identities (15) and (16),

$$
\begin{aligned}
{\left[(a b)^{J}-\right.} & \left.a^{J} b^{J}, c^{J} d^{J}\right] \\
= & (a b c d+d c a b)^{J}-(c d+d c)^{J}(a b)^{J}-a^{J}(b c d+d c b)^{J}+(a d c+c d a)^{J} b^{J} \\
= & (a b c d)^{J}-a^{J}(b c d)^{J}+(d c a b)^{J}-(d c)^{J}(a b)^{J} \\
& +(a d c b)^{J}-a^{J}(d c b)^{J}-\left[(a d c b)^{J}-(a d c)^{J} b^{J}\right] \\
& +(c d a b)^{J}-(c d)^{J}(a b)^{J}-\left[(c d a b)^{J}-(c d a)^{J} b^{J}\right] .
\end{aligned}
$$

It follows that $\mathfrak{N}^{+}$is a Lie ideal in $\mathfrak{N}$ and a similar proof shows that $\mathfrak{N}^{-}$is also a Lie ideal in $\mathfrak{R}$.

We have

$$
\begin{aligned}
{\left[\left[(a b)^{J}-a^{J} b^{J},(c d)^{J}-d^{J} c^{J}\right] x^{J}\right]=} & {\left[\left[(a b)^{J},(c d)^{J}\right] x^{J}\right]+\left[\left[d^{J} c^{J},(a b)^{J}\right] x^{J}\right] } \\
& +\left[\left[(c d)^{J}, a^{J} b^{J}\right] x^{J}\right]+\left[\left[a^{J} b^{J}, d^{J} c^{J}\right], x^{J}\right]
\end{aligned}
$$

Each term on the right-hand side of (19) will be considered individually. The first term can be written

$$
\left[\left[(a b)^{J},(c d)^{J}\right] x^{J}\right]=(a b c d x-c d a b x-x a b c d+x c d a b)^{J} .
$$

After application of (17), the second and third terms become

$$
\begin{aligned}
{\left[\left[d^{J} c^{J},(a b)^{J}\right] x^{J}\right]=} & -(a b c d x+x d c a b-c d a b x-x a b d c)^{J} \\
& -(a b d c+c d a b-a b c d-d c a b)^{J} x^{J}, \\
{\left[\left[(c d)^{J}, a^{J} b^{J}\right], x^{J}\right]=} & (c d b a x+x a b c d-b a c d x-x c d a b)^{J} \\
& +(c d a b+b a c d-c d b a-a b c d)^{J} x^{J} .
\end{aligned}
$$

By (18) the last term becomes

$$
\begin{aligned}
{\left[\left[a^{J} b^{J}, d^{J} c^{J}\right], x^{J}\right]=} & (x d c a b+b a c d x-x a b d c-c d b a x)^{J} \\
& +(a b d c+c d b a-d c a b-b a c d)^{J} x^{J}
\end{aligned}
$$

Substitution of (20)-(23) in (19) gives zero. Therefore $\left[\mathfrak{N}^{+}, \mathfrak{N}^{-}\right] \subseteq \subseteq$.

A simple adaptation of the argument in the proof of Theorem 16 gives the following theorem.

Theorem 18. Let $J$ be a Jordan homomorphism of a ring $\mathfrak{A}$ into a ring $\mathfrak{B}$ 
and denote by $\mathfrak{R}$ the extended enveloping Lie ring of $\mathfrak{U}^{J}$ and by $\mathfrak{\Im}$ the center of $\mathfrak{\Re}$. Assume (i) $\mathfrak{\Re} / \mathfrak{S}$ contains no commutative Lie ideals, (ii) any two Lie ideals in $\mathfrak{N} / \subseteq$ Save a nonzero intersection, and (iii) no nonzero element of $\mathfrak{S}$ is a zero divisor in $\mathfrak{N}$. Then $J$ is either a homomorphism or anti-homomorphism.

8. Lie structure of matrix rings. Application to primitive rings with minimal ideals. In order to apply the results of the preceding two sections to the case of primitive rings with minimal ideals, some information concerning the ideal structure of the associated special Lie rings is needed. We consider first a general matrix ring $\mathfrak{D}_{n}$, where $n \geqq 2$ and, in case $n=2,2 \mathfrak{D}=\mathfrak{D}$.

THEOREM 19. Let $\mathfrak{J}$ be any Lie ideal in $\mathfrak{D}_{n}$ not contained in the center of $\mathfrak{D}_{n}$. Then there exists a nonzero ideal $\Lambda$ in the ring $\mathfrak{D}$ such that $\left[\mathfrak{D}_{n}, \Lambda_{n}\right] \subseteq \mathfrak{J}$.

Proof. Denote by $\Lambda$ the collection of all $\alpha \in \mathfrak{D}$ such that $\alpha e_{i j} \in J$ for some $i \neq j$. We prove that $\Lambda_{n}$ satisfies the desired conditions.

First let $\alpha$ be an arbitrary element of $\Lambda$ and choose $i \neq j$ such that $\alpha e_{i j} \in J$. We show that $\alpha e_{p q} \in J$ for all $p \neq q$. If $n \geqq 3$, choose $k$ such that $k \neq i$ and $k \neq j$. Then

$$
\alpha e_{p q}=\left[e_{k q}\left[e_{p i}\left[e_{j k}, \alpha e_{i j}\right]\right]\right]
$$

and hence $\alpha e_{p q} \in \mathfrak{J}$. On the other hand, if $n=2$, then $2 \mathfrak{D}=\mathfrak{D}$ and there exists $\omega \in \mathfrak{D}$ such that $2 \omega=1$. Moreover

$$
\alpha e_{j i}=\left[\omega e_{j j}\left[e_{j i}\left[\alpha e_{i j}, e_{j i}\right]\right]\right]
$$

and hence $\alpha e_{j i} \in J$. It follows that $\alpha e_{p q} \in J$ for all $p \neq q$ in this case as well. We can now conclude that $\Lambda$ is a group under addition. Moreover, for $\alpha \in \Lambda$ and $d \in \mathfrak{D}, d \alpha e_{i j}=\left[d e_{i i}, \alpha e_{i j}\right]$ and $\alpha d e_{i j}=\left[\alpha e_{i j}, d e_{j j}\right]$ for $i \neq j$. Therefore $\Lambda$ is an ideal in $\mathfrak{D}$ and hence $\Lambda_{n}$ is an ideal in $\mathfrak{D}_{n}$. We prove next that $\Lambda_{n} \neq(0)$. Let $a=\sum \alpha_{i j} e_{i j}$ be an element of $\mathfrak{J}$ not in the center of $\mathfrak{D}_{n}$ and assume first that $\alpha_{i j}=0$ for all $i \neq j$. Then $a=\sum \alpha_{i i} e_{i i}$. Since $a$ is not in the center of $\mathfrak{D}_{n}$, there exists $d e_{r s}, d \in \mathfrak{D}$, such that $\left[a, d e_{r s}\right]=\left(\alpha_{r r} d-d \alpha_{s s}\right) e_{r s} \neq 0$. If $r \neq s$ it is immediate that $\Lambda_{n} \neq(0)$. If $r=s$, choose $t \neq r$. Then $\left[\alpha_{r r}, d\right] \neq 0$ and

$$
\left[\left[a, d e_{r r}\right] e_{r t}\right]=\left[\alpha_{r r}, d\right] e_{r t}
$$

and again $\Lambda_{n} \neq(0)$. Now assume that $\alpha_{i j} \neq 0$ for some $i \neq j$. If $n \geqq 3$, there exists $k$ such that $k \neq i$ and $k \neq j$. Thus $\left[e_{j i}\left[e_{j k}\left[a, e_{i i}\right]\right]\right]=\alpha_{i j} e_{j k}$ so that $\alpha_{i j} \in \Lambda$. If $n=2$, choose $\omega \in \mathfrak{D}$ as before so that $2 \omega=1$. Then

$$
\left[\omega e_{j i}\left[e_{j i}\left[a e_{j i}\right]\right]\right]=\alpha_{i j} e_{j i} \text {. }
$$

$\Lambda_{n} \neq(0)$ in this case as well.

In order to prove that $\left[\mathfrak{D}_{n}, \Lambda_{n}\right] \subseteq J$, it will be sufficient to prove that $\left[d e_{i j}, \alpha e_{p q}\right] \in J$ for arbitrary $i, j, p, q$ and $d \in \mathfrak{D}, \alpha \in \Lambda$. Since $\alpha e_{p q} \in \mathfrak{J}$ for $p \neq q$, we can assume $p=q$. Then $\left[d e_{i j}, \alpha e_{p p}\right]=d \alpha \delta_{j p} e_{i p}-\alpha d \delta_{p i} e_{p j}$. If either $i \neq p$ or 
$j \neq p$, it is immediate that $\left[d e_{i j}, \alpha e_{p p}\right] \in J$. Hence assume $i=p=j$. Then

Since

$$
\left[d e_{i j}, \alpha e_{p p}\right]=[d \alpha] e_{p p} \text {. }
$$

$$
[d \alpha] e_{p p}=\left[d e_{p p}\left[\alpha e_{p q}, e_{q p}\right]\right]
$$

and $\alpha e_{p q} \in J$ for $p \neq \neq q$, it follows that $[d \alpha] e_{p p} \in J$. This completes the proof.

Observe that if the matrix ring $\mathfrak{D}_{n}$ is simple, then every Lie ideal of $\mathfrak{D}_{n}$, not contained in the center of $\mathfrak{D}_{n}$, must contain $\left[\mathfrak{D}_{n}, \mathfrak{D}_{n}\right]$. More generally, if $\mathfrak{A}$ is a locally matrix ring such that every finite subset of $\mathfrak{A}$ can be contained in a simple matrix ring, then every Lie ideal of $\mathfrak{A}$, not in the center of $\mathfrak{A}$,

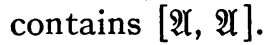

In particular this is true if $\mathfrak{A}$ is a simple ring with minimal ideals.

In the remainder of this section $\mathfrak{B}$ will denote a primitive ring with minimal ideals. The notations introduced in $\$ 5$ will be used here. It will be assumed throughout our discussion that $\operatorname{dim} \mathfrak{X} \geqq 2$ and, if $\operatorname{dim} \mathfrak{X}=2$, then $\Delta$ is not of characteristic two.

THEOREM 20. Every Lie ideal in $\mathfrak{B}$ not contained in the center of $\mathfrak{B}$ contains $[\mathfrak{F}, \mathfrak{F}]$.

Proof. If $\mathfrak{X}$ is finite-dimensional, then $\mathscr{B}=\mathfrak{F}$ and the desired result follows from Theorem 19. Therefore assume $\mathfrak{X}$ to be infinite-dimensional in which case $\mathfrak{F}$ has center zero. Let $\mathfrak{J}$ be a Lie ideal in $\mathfrak{B}$ not contained in the center of $\mathfrak{B}$ and set $\mathfrak{J}_{0}=\mathfrak{J} \cap \mathfrak{F}$. Since the centralizer of $\mathfrak{F}$ in $\mathfrak{B}$ is equal to the center of $\mathfrak{B}$, there exist elements $f \in \mathfrak{F}$ and $a \in \mathfrak{J}$ such that $[f, a] \neq 0$. Evidently $[f, a] \in \mathfrak{J} \cap \mathfrak{F}$ so that $\mathfrak{J}_{0} \neq(0)$. Since $\mathfrak{F}$ is simple and $\mathfrak{J}_{0}$ is a nonzero Lie ideal in $\mathfrak{F}$, it follows that $[\mathfrak{F}, \mathfrak{F}] \subseteq \mathfrak{J}_{0} \subseteq \mathfrak{J}$, by the remark following the proof of Theorem 19.

By virtue of Theorem 20 we can apply Theorem 18 to the case of primitive rings. In what follows $\mathfrak{B}$ will denote a primitive ring satisfying the conditions imposed for Theorem 20.

THEOREM 21. Let $J$ be any Jordan homomorphism of a ring $\mathfrak{A}$ into the ring $\mathfrak{B}$. If the extended enveloping Lie ring of $\mathfrak{H}^{J}$ is equal to $\mathfrak{B}$, then $J$ is either a homomorphism or an anti-homomorphism.

Proof. We have only to prove that $\mathfrak{R}=\mathfrak{B}$ satisfies conditions (i)-(iii) of Theorem 18. Let $\overline{\mathfrak{J}}$ denote any nonzero Lie ideal in $\mathfrak{B} / \mathfrak{S}$, where $\mathfrak{S}$ is the center of $\mathfrak{B}$. The union of all cosets belonging to $J$ is clearly a Lie ideal in $\mathfrak{B}$ not contained in $\mathfrak{S}$. Therefore, by Theorem $20,[\mathfrak{F}, \mathfrak{F}] \subseteq \mathfrak{J}$ and hence $([\mathfrak{F}, \mathfrak{F}]$ $+\mathfrak{S}) / \mathfrak{S} \subseteq \bar{\jmath}$. Since $([\mathfrak{F}, \mathfrak{F}]+\mathfrak{S}) / \mathfrak{S}$ is evidently a noncommutative ideal in $\mathfrak{B} / \mathfrak{S}$, it follows that conditions (i) and (ii) are satisfied. Since $\mathfrak{S}$ is contained in $\Delta$, condition (iii) is also satisfied.

CoRollary. Any Jordan homomorphism of a ring $\mathfrak{A}$ onto the primitive ring 
$\mathfrak{B}$ is either a homomorphism or an anti-homomorphism.

9. Some examples. In the preceding section we have solved the problem of determining the Jordan homomorphisms of rings onto primitive rings with minimal ideals. However, this does not give a complete solution of the problem of Jordan homomorphisms of primitive rings with minimal ideals. For certain rings of this type the results of $\$ 4$ give a complete solution. On the other hand, as we proceed to show by examples, Jordan homomorphisms of primitive rings may be more complicated than the sums of homomorphisms and anti-homomorphisms.

We consider first the following rings which are not primitive.

EXAmple 3 . Let $\mathfrak{A}$ and $\mathfrak{B}$ be algebras with bases $(e, f, g)$ and $\left(e^{\prime}, f^{\prime}, g^{\prime}\right)$ respectively and multiplication tables as follows:

\begin{tabular}{l|lll} 
& $e$ & $f$ & $g$ \\
\hline$e$ & $e$ & $e$ & $e$ \\
$f$ & $f$ & $f$ & $f$ \\
$g$ & $g$ & $g$ & $g$
\end{tabular}

\begin{tabular}{c|ccc} 
& $e^{\prime}$ & $f^{\prime}$ & $g^{\prime}$ \\
\hline$e^{\prime}$ & $e^{\prime}$ & $g^{\prime}$ & $g^{\prime}$ \\
$f^{\prime}$ & $e^{\prime}+f^{\prime}-g^{\prime}$ & $f^{\prime}$ & $f^{\prime}$ \\
$g^{\prime}$ & $e^{\prime}$ & $g^{\prime}$ & $g^{\prime}$
\end{tabular}

Then $e^{2}=e, f^{2}=f, g^{2}=g,\{e f\}=e+f,\{e g\}=e+g,\{f g\}=f+g$ and $\left(e^{\prime}\right)^{2}=e^{\prime}$, $\left(f^{\prime}\right)^{2}=f^{\prime}, \quad\left(g^{\prime}\right)^{2}=g^{\prime}, \quad\left\{e^{\prime} f^{\prime}\right\}=e^{\prime}+f^{\prime}, \quad\left\{e^{\prime} g^{\prime}\right\}=e^{\prime}+g^{\prime},\left\{f^{\prime} g^{\prime}\right\}=f^{\prime}+g^{\prime}$. Hence $\alpha e$ $+\beta f+\gamma g \rightarrow \alpha e^{\prime}+\beta f^{\prime}+\gamma g^{\prime}$ is a Jordan isomorphism which is neither an isomorphism nor an anti-isomorphism.

EXAmple 4. Let $\$$ be a subalgebra of a matrix algebra $\Phi_{r}$ which has a Jordan homomorphism that is not a homomorphism or anti-homomorphism. Thus we can take $\mathbb{B}$ to be a matrix algebra which is isomorphic to the $\mathfrak{A}$ of example 3. Let $\mathfrak{A}$ be the algebra of infinite matrices of the form

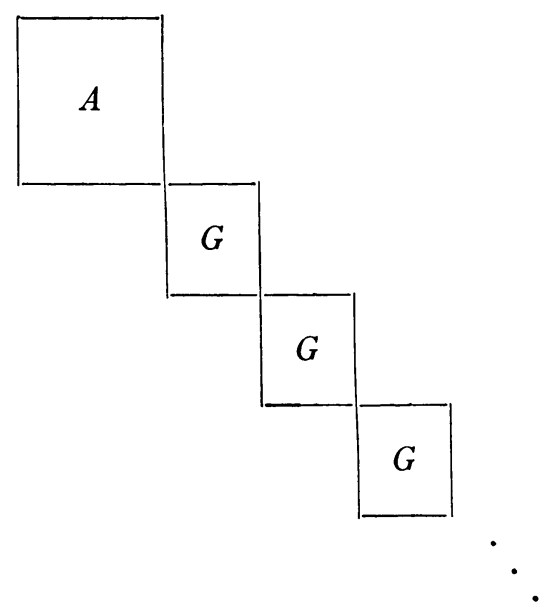

where $A \in \Phi_{n r}, n$ arbitrary, and $G \in \mathfrak{S}$. Then $\mathfrak{A}$ contains the matrices of the 
form (24) with $G=0$ and the linear transformations corresponding to these matrices have finite rank. It follows easily that $\mathfrak{A}$ is a primitive ring with minimal ideals. Also it is clear that the mapping which sends the matrix (24) into $G$ is a homomorphism. If we follow this mapping with a Jordan homomorphism of $B$ which is not a homomorphism or anti-homomorphism, then we obtain a Jordan homomorphism of $\mathfrak{A}$ which is neither a homomorphism nor an anti-homomorphism.

10. Application to derivations. An additive mapping $D$ of a ring $\mathfrak{A}$ into itself is called a derivation provided

$$
(a b)^{D}=a b^{D}+a^{D} b .
$$

It is called a Jordan derivation provided

$$
\left(a^{2}\right)^{D}=a a^{D}+a^{D} a
$$

and also

$$
(a b a)^{D}=a^{D} b a+a b^{D} a+a b a^{D} .
$$

It follows from (26) that

$$
\{a b\}^{D}=\left\{a b^{D}\right\}+\left\{a^{D} b\right\},
$$

where $\{a b\}=a b+b a$. If no element of $\mathfrak{A}$ is of additive order two, then it is not difficult to show that (28) implies (26) and (27). It is obvious that every derivation is a Jordan derivation. Our purpose here is to show that, for certain rings $\mathfrak{A}$, every Jordan derivation is actually a derivation.

THEOREM 22. If $\mathfrak{A}$ has an identity and if every Jordan homomorphism of $\mathfrak{A}$ is the sum of a homomorphism and an anti-homomorphism, then every Jordan derivation in $\mathfrak{A}$ is a derivation.

Proof. Define the mapping $J$ of $\mathfrak{A}$ by

$$
a \rightarrow a^{J}=\left(\begin{array}{ll}
a & a^{D} \\
0 & a
\end{array}\right) .
$$

Then $J$ is a Jordan homomorphism of $\mathfrak{A}$. Denote the enveloping ring of $\mathfrak{A}^{J}$ by $\mathbb{E}$ and note that $\mathbb{E}$ has an identity since $\mathfrak{A}$ has. By hypothesis, $J$ is the sum of a homomorphism and an anti-homomorphism. Hence there exists a decomposition $1=E_{1}+E_{2}$ of the identity in $\&$ such that $E_{1}, E_{2}$ are in the center of $\mathcal{E}, E_{i} E_{j}=\delta_{i j} E_{i}, a \rightarrow a^{J} E_{1}$ is a homomorphism and $a \rightarrow a^{J} E_{2}$ is an anti-homomorphism. If

$$
E_{i}=\left(\begin{array}{ll}
e_{i} & u_{i} \\
0 & e_{i}
\end{array}\right)
$$$$
i=1,2 \text {, }
$$

then it is clear that $1=e_{1}+e_{2}, e_{i} e_{j}=\delta_{i j} e_{i}$, and $e_{1}, e_{2}$ are in the center of $\mathfrak{A}$. 
Using the fact that $a \rightarrow a^{J} E_{1}$ is a homomorphism, we obtain

$$
\begin{aligned}
\left(\begin{array}{cc}
a b & (a b)^{D} \\
0 & a b
\end{array}\right)\left(\begin{array}{cc}
e_{1} & u_{1} \\
0 & e_{1}
\end{array}\right) & =\left(\begin{array}{cc}
a & a^{D} \\
0 & a
\end{array}\right)\left(\begin{array}{ll}
b & b^{D} \\
0 & b
\end{array}\right)\left(\begin{array}{ll}
e_{1} & u_{1} \\
0 & e_{1}
\end{array}\right) \\
& =\left(\begin{array}{cc}
a b e_{1} & a b u_{1}+\left(a b^{D}+a^{D} b\right) e_{1} \\
0 & a b e_{1}
\end{array}\right) .
\end{aligned}
$$

Therefore

$$
a b u_{1}+(a b)^{D} e_{1}=a b u_{1}+\left(a b^{D}+a^{D} b\right) e_{1}
$$

or

$$
(a b)^{D} e_{1}=\left(a b^{D}+a^{D} b\right) e_{1}
$$

Similarly, since $a \rightarrow a^{J} E_{2}$ is an anti-homomorphism,

$$
\begin{aligned}
\left(\begin{array}{cc}
a b & (a b)^{D} \\
0 & a b
\end{array}\right)\left(\begin{array}{cc}
e_{2} & u_{2} \\
0 & e_{2}
\end{array}\right) & =\left(\begin{array}{cc}
b & b^{D} \\
0 & b
\end{array}\right)\left(\begin{array}{cc}
a & a^{D} \\
0 & a
\end{array}\right)\left(\begin{array}{cc}
e_{2} & u_{2} \\
0 & e_{2}
\end{array}\right) \\
& =\left(\begin{array}{cc}
b a e_{2} & b a u_{2}+\left(b a^{D}+b^{D} a\right) e_{2} \\
0 & b a e_{2}
\end{array}\right) .
\end{aligned}
$$

Therefore

$$
a b e_{2}=b a e_{2}
$$

and

$$
a b u_{2}+(a b)^{D} e_{2}=b a u_{2}+\left(b a^{D}+b^{D} a\right) e_{2} .
$$

Multiplying (31) on the right by $e_{2}$ and observing that, by (30), bau $e_{2}$ $=a b u_{2} e_{2}$ and $\left(b a^{D}+b^{D} a\right) e_{2}=\left(a b^{D}+a^{D} b\right) e_{2}$, we obtain

$$
(a b)^{D} e_{2}=\left(a b^{D}+a^{D} b\right) e_{2}
$$

Addition of (29) and (32), with the fact that $1=e_{1}+e_{2}$, gives $(a b)^{D}=a b^{D}+a^{D} b$, that is, $D$ is a derivation.

\section{BIBLIOGRAPHY}

1. G. Ancochea, Le Théorème de von Staudt en géométrie projective quaternionienne, Journal für Mathematik vol. 184 (1942) pp. 192-198.

2. - On semi-automorphisms of division algebras, Ann. of Math. vol. 48 (1947) pp. 147-154.

3. G. Birkhoff and P. M. Whitman, Representation of Jordan and Lie algebras, Trans. Amer. Math. Soc. vol. 65 (1949) pp. 116-136.

4. J. Dieudonne, Sur le socle d'un anneau et les anneaux simples infinis, Bull. Soc. Math. France vol. 71 (1943) pp. 1-30.

5. L. K. Hua, On the automorphisms of a field, Proc. Nat. Acad. Sci. U.S.A. vol. 35 (1949) pp. $386-389$. 
6. N. Jacobson, Theory of rings, Mathematical Surveys, vol. 2, New York, American Mathematical Society, 1943.

7. - Structure theory of simple rings without finiteness assumptions. Trans. Amer. Math. Soc. vol. 57 (1945) pp. 228-245.

8. - On the theory of primitive rings, Ann. of Math. vol. 48 (1947) pp. 8-21.

9. - Isomorphisms of Jordan rings, Amer. J. Math. vol. 70 (1948) pp. 317-326.

10. - Lie and Jordan triple systems, Amer. J. Math. vol. 71 (1948) pp. 149-170.

11. F. D. Jacobson and N. Jacobson, Classification and representation of semi-simple Jordan algebras, Trans. Amer. Math. Soc. vol. 65 (1949) pp. 141-169.

12. P. Jordan, Über eine Klasse nichtassoziativer hyperkomplexer Algebren, Nachr. Ges. Wiss. Göttingen (1932) pp. 569-575. 527.

13. I. Kaplansky, Semi-automorphisms of rings, Duke Math. J. vol. 14 (1947) pp. 521-

YaLE UNIVERSITY,

New Haven, Conn. 